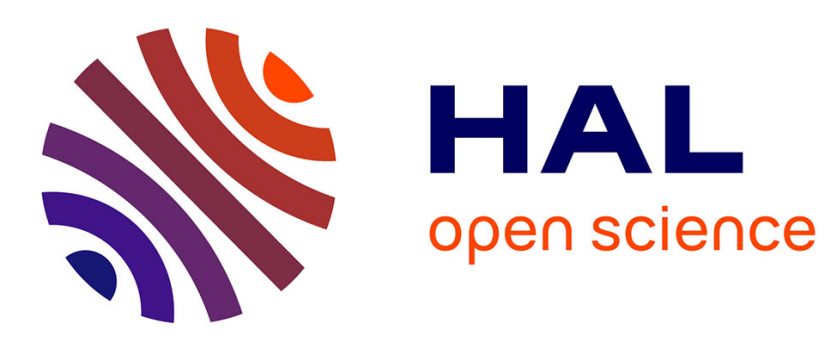

\title{
Large-eddy simulation of a bi-periodic turbulent flow with effusion
}

\author{
Simon Mendez, Franck Nicoud
}

\section{To cite this version:}

Simon Mendez, Franck Nicoud. Large-eddy simulation of a bi-periodic turbulent flow with effusion. Journal of Fluid Mechanics, 2008, 598, pp.27-65. 10.1017/S0022112007009664 . hal-00820464

\section{HAL Id: hal-00820464 https://hal.science/hal-00820464}

Submitted on 5 May 2013

HAL is a multi-disciplinary open access archive for the deposit and dissemination of scientific research documents, whether they are published or not. The documents may come from teaching and research institutions in France or abroad, or from public or private research centers.
L'archive ouverte pluridisciplinaire HAL, est destinée au dépôt et à la diffusion de documents scientifiques de niveau recherche, publiés ou non, émanant des établissements d'enseignement et de recherche français ou étrangers, des laboratoires publics ou privés. 


\title{
Large-eddy simulation of a bi-periodic turbulent flow with effusion
}

\author{
By S. MENDEZ AND F. NICOUD $\mathbf{Z}^{2} \dagger$ \\ ${ }^{1}$ CERFACS - 42, Av. Gaspard Coriolis, 31057 Toulouse cedex 1 - France. \\ ${ }^{1,2}$ University Montpellier II - I3M CNRS UMR 5149 \\ Place Eugène Bataillon. 34095 Montpellier cedex 5 - France.
}

(Received 22 December 2006, and in revised form 5 September 2007)

Large-Eddy Simulations of a generic turbulent flow with discrete effusion are reported. The computational domain is periodic in both streamwise and spanwise directions and contains both the injection and the suction sides. The blowing ratio is close to 1.2 while the Reynolds number in the aperture is of order 2600. The numerical results for this fully developed, bi-periodic turbulent flow with effusion are compared to available experimental data from a large-scale, spatially evolving isothermal configuration. It is shown that many features are shared by the two flow configurations. The main difference is related to the mean streamwise velocity profile that is more flat for the bi-periodic situation where the cumulative effect of an infinite number of upstream jets is accounted for. The necessity of considering both sides of the plate is also established by analysing the vortical structure of the flow and some differences with the classical jet-in-crossflow case are highlighted. Eventually, the numerical results are analysed in terms of wall modelling for full-coverage film cooling. For the operating point considered, it is demonstrated that the streamwise momentum flux is dominated by non-viscous effects, although the area where only the viscous shear stress contributes is very large given the small porosity value $(4 \%)$.

\section{Introduction and objectives}

In gas turbines, the solid parts such as the turbine blades or the liner of the combustion chamber are submitted to large thermal constraints and must be cooled. As pointed out by Lefebvre (1999), the most efficient cooling system is transpiration-based: the solid parts to be cooled are made of porous material through which cool air is injected. The resulting uniform film of fresh gas isolates the solid parts from the hot products. However, the application of transpiration-based technology to gas turbines is impossible because of the mechanical weakness of available porous materials and alternative solutions are sought for. One possibility, widely employed for combustion chamber walls, is to use multi-perforated walls to produce the necessary cooling. In this approach (see figure 1), fresh air coming from the casing goes through angled perforations and enters the combustion chamber. The generated micro-jets coalesce to form a film that protects the liner from the hot gases. This technique is usually called full-coverage film cooling (FCFC) to distinguish it from the film cooling (FC) system used for turbine blades, where only a few cooling holes are needed. FCFC corresponds to a discrete form of the transpiration cooling approach.

$\dagger$ Corresponding author: franck.nicoud@univ-montp2.fr 
COMBUSTION CHAMBER: injection side

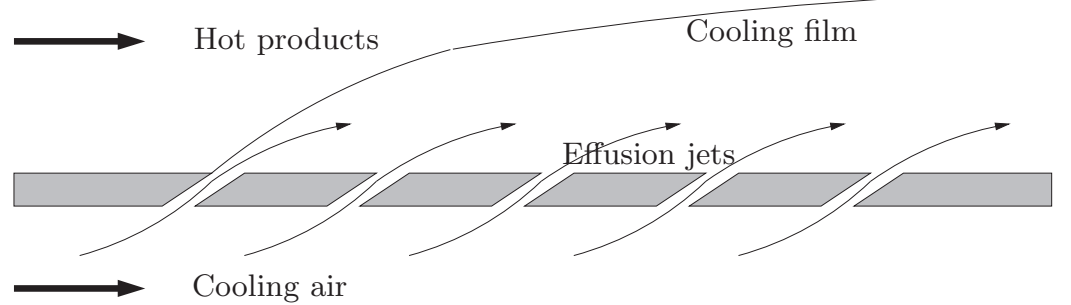

CASING: suction side

FIgURE 1. Principle of full-coverage film cooling: fresh air flowing in the casing is injected into the combustion chamber through the liner perforations and forms an isolating film protecting the internal face of the liner from the combustion gases.

When wall cooling is ensured by FCFC, the number of submillimetric holes is large and does not allow a complete description of the generation and coalescence of the jets when computing the 3-D turbulent reacting flow within the burner. Effusion is however known to have drastic effects on the whole flow structure, notably by changing the flame position and subsequently modifying the temperature field. An appropriate model is thus needed to reproduce the effect of effusion cooling on the main flow. Such a modelling has already been done for transpired boundary layers and extended law-of-the-wall for moderate uniform blowing or suction is available (Piomelli et al. 1989; Simpson 1970). However, existing models accounting for moderate transpiration can hardly been adapted to FCFC. It is quite obvious that for a given mass flow rate per unit area $\dot{m}$, the injected momentum flux per unit area is different depending on the type of injection: it will be $\dot{m}^{2} / \rho$ (with $\rho$ the mass density of the injected fluid) in the case of a uniform injection whereas of order $\dot{m}^{2} / \rho \sigma$ if the injection is through a multi-perforated plate of porosity $\sigma$ (hole-to-total surface ratio). As a consequence, new wall models for turbulent flows with effusion are required to perform predictive full-scale computations. Note also that for practical reasons, existing models are essentially local in space: they allow the assessment of the fluxes through a (solid) boundary at a given position based on the knowledge of the outer flow conditions right above that same position. For example, when computing a spatially evolving boundary layer at high-Reynolds number, a Reynolds-Averaged NavierStokes (RANS) approach will use the classical logarithmic law-of-the-wall to evaluate the local wall shear stress based on the tangential velocity at the first off-wall grid point or cell centre. This law-of-the-wall is local in the sense that the knowledge of the distance from the leading edge is not required for assessing the wall shear stress. To be useful in practical RANS computations, any FCFC model should meet the same property and relate the fluxes through the effusion plate at a given position to the outer flow quantities at the same position, on both the suction and the injection sides. Note that despite the numerous studies dealing with FCFC and FC, data relating wall fluxes to suction and injection quantities are unusual.

Tables 1 and 2 give an overview of the main experimental (table 1) and numerical (table 2) studies related to injection/suction through perforated plates: Jet in Cross Flow (JCF); one row of holes (FC) or several rows of holes (FCFC). JCF references are included because in the combustion chamber side of the liner, the cooling film arising from FCFC is generated by hundreds of tiny JCF. Note however that the FCFC jets differ 


\begin{tabular}{|c|c|c|}
\hline & $\begin{array}{l}\text { Isothermal } \\
\text { Flow measurements }\end{array}$ & $\begin{array}{l}\text { Non-isothermal } \\
\text { Wall data }\end{array}$ \\
\hline JCF and FC & $\begin{array}{c}\text { Andreopoulos \& Rodi (1984) (I) } \\
\text { Pietrzyk et al. (1989) (I) } \\
\text { Fric \& Roshko (1994)(I) } \\
\text { Kelso et al. (1996)(I) } \\
\text { Smith \& Mungal (1998) (I) } \\
\text { MacManus \& Eaton (2000) (S) } \\
\text { Peterson \& Plesniak }(2004 a)(\mathrm{S} / \mathrm{H} / \mathrm{I})\end{array}$ & $\begin{array}{l}\text { Goldstein (1971) (I) } \\
\text { Eriksen \& Goldstein (1974) (I) } \\
\text { Ammari et al. }(1990) \text { (I) }\end{array}$ \\
\hline FCFC & $\begin{array}{c}\text { Yavuzkurt et al. }(1980 a, b) \text { (I) } \\
\text { Gustafsson (2001) (I) } \\
\text { Miron (2005) (I) }\end{array}$ & $\begin{array}{c}\text { Metzger et al. }(1973)(\mathrm{I}) \\
\text { Mayle \& Camarata (1975) (I) } \\
\text { Crawford et al. }(1980)(\mathrm{I}) \\
\text { Cho \& Goldstein }(1995 a, b)(\mathrm{S} / \mathrm{H} / \mathrm{I}) \\
\text { Rouvreau }(2001)(\mathrm{I}) \\
\text { Dorignac et al. }(2005)(\mathrm{S})\end{array}$ \\
\hline \multicolumn{3}{|c|}{$\begin{array}{l}\text { TABLE 1. Major experimental studies concerning jets in crossflow (JCF), film cooling (FC) } \\
\text { and full-coverage film cooling (FCFC), depending on if the injection (I), the suction (S) or } \\
\text { the hole (H) are studied. The classification depends on the thermal characteristics (isother- } \\
\text { mal/non-isothermal) and on the type of data provided. Flow measurements: spatially re- } \\
\text { solved data is provided, e.g. temperature, velocity or vorticity fields and profiles; Wall data: } \\
\text { integrated or local wall data is provided, e.g. adiabatic or overall cooling effectiveness, or heat } \\
\text { transfer coefficient. Note that no references were found with flow measurements in a non-isother- } \\
\text { mal case. }\end{array}$} \\
\hline
\end{tabular}

\begin{tabular}{|c|c|c|}
\hline \multirow[b]{2}{*}{$\mathrm{JCF}$ and FC } & Isothermal & Non-isothermal \\
\hline & $\begin{array}{l}\text { Yuan et al. }(1999)(\mathrm{I}) \\
\text { Schlüter \& Schönfeld (2000) (I) } \\
\text { Cortelezzi \& Karagozian (2001) (I) } \\
\text { Prière et al. }(2005)(\mathrm{I}) \\
\text { MacManus \& Eaton }(2000) \text { (S) }\end{array}$ & $\begin{array}{l}\text { Walters \& Leylek }(2000)(\mathrm{H} / \mathrm{I}) \\
\text { Tyagi \& Acharya }(2003)(\mathrm{H} / \mathrm{I}) \\
\text { Renze et al. }(2006)(\mathrm{H} / \mathrm{I}) \\
\text { Iourokina \& Lele }(2006)(\mathrm{S} / \mathrm{H} / \mathrm{I}) \\
\text { Peet }(2006)(\mathrm{S} / \mathrm{H} / \mathrm{I})\end{array}$ \\
\hline FCFC & no reference found & $\begin{array}{l}\text { Papanicolaou et al. (2001) (I) } \\
\text { Harrington et al. (2001) (I) }\end{array}$ \\
\hline \multicolumn{3}{|c|}{$\begin{array}{l}\text { TABLE 2. Major numerical studies concerning jets in crossflow (JCF), film cooling (FC) and full- } \\
\text { coverage film cooling (FCFC), depending on if the injection (I), the suction (S) or the hole }(\mathrm{H}) \text { are } \\
\text { studied. The classification depends on the thermal characteristics (isothermal/non-isothermal) } \\
\text { of the configuration. }\end{array}$} \\
\hline
\end{tabular}

from the canonical configurations of JCF in several aspects (see the review by Margason (1993) for more details about canonical JCF):

(a) While single canonical JCF is usually designed to penetrate in the main flow and enhance mixing, the purpose of effusion jets is to create a film to protect the wall from the hot gases. Many jets are used to form the film, and they are oriented so that cooling air stays next to the wall, without mixing with the main flow. Note that both configurations are encountered in gas turbines: contrary to the small cooling jets that protect the wall, JCF called dilution jets are designed to penetrate the combustion chamber to dilute the combustion products in the secondary zone,

(b) In FCFC, the crossflow is not a simple boundary layer as for JCF studies but results from the interaction between all the jets located upstream, 
(c) The inclination of the jets in FCFC application is smaller than in JCF, modifying the penetration of the jets as well as the interaction with the main flow (see for example Bergeles, Gosman \& Launder 1976, 1977),

(d) Because of the small length-to-diameter ratio of the holes used in FCFC applications, the flow on the injection side is strongly related to the flow in the aperture and on the suction side (Iourokina \& Lele 2006; Peterson \& Plesniak 2004a; Walters \& Leylek 2000). This is particularly striking in studies about discharge coefficient, where the velocity in the supply channel is one of the main parameters (Champion et al. 2005; Gritsch et al. 2001).

In views of these differences, extrapolating the results from canonical JCF studies to gain insight into FCFC would not be justified and specific FCFC configurations must be considered. This is in line with Walters \& Leylek (2000) who insist on the importance of reproducing the exact geometry for film cooling studies. The aim of this study is to generate detailed data numerically and to reach a better understanding of turbulent flows with effusion, a first step towards the development of appropriate wall models for FCFC.

Ideally, relevant data to build FCFC models would contain detailed information about the dynamical and thermal behaviours of the flow on both sides of the plate and would correspond to a realistic FCFC configuration, viz. with a large array of holes submitted to non-isothermal flow conditions. From tables 1 and 2, such data does not exist. Detailed flow measurements in non-isothermal situations have never been performed and the complete configuration (suction side/hole/injection side, as in Cho \& Goldstein 1995a,b) has been rarely considered. Only Peet (2006), Iourokina \& Lele (2006) and Peterson \& Plesniak (2004a) detail the flow on the suction side, in the aperture and on the injection side. However, only one row of holes is considered and all the flow on the suction side is forced through the holes, as in FC applications. Few studies concern the suction side of the plate: MacManus \& Eaton (2000) treat the suction of a laminar boundary layer by an isolated hole and a small array of holes, in an isothermal configuration. Dorignac et al. (2005) propose global measurements and correlations of the Nusselt number on the suction side of a multi-perforated plate. However, the aspiration is performed from a medium at rest, which is not representative of the real conditions in the casing of a gas turbine.

Most of the experimental studies concerning discrete-hole film cooling address the case of a single row of holes (cooling application for turbine blades) so that only few studies dealing with several rows configurations are available. All the spatially resolved aerodynamic measurements available correspond to large-scale isothermal flows (Gustafsson 2001; Miron 2005; Yavuzkurt, Moffat \& Kays 1980a,b). Moreover, experimental studies addressing the thermal behaviour (evaluation of cooling effectiveness or heat transfer coefficient at the wall) do not provide any flow measurements (Cho \& Goldstein 1995a,b; Crawford, Kays \& Moffat 1980; Mayle \& Camarata 1975; Metzger, Takeuchi \& Kuenstler 1973) or insufficiently resolved ones (Rouvreau 2001). Note however the very interesting work by Cho \& Goldstein $(1995 a, b)$, who estimated the local wall heat transfer on the back surface (suction side), the holes and the exposed surface (injection side) on both $\mathrm{FC}$ and FCFC configurations.

Numerical capabilities have increased during the last years and RANS simulations of FCFC with several rows (7 for Papanicolaou et al. (2001), 10 for Harrington et al. (2001) and 12 rows for Errera \& Chemin (2004)) have been performed. These simulations prove the ability of numerical codes to reproduce effusion flows. However, the idea of designing wall models by using RANS based data remains questionable. As long as only a few numbers of holes are considered, Large-Eddy Simulations (LES) can be performed in place of RANS calculations in order to gain insight into the jet-mainstream interaction. Recently, 
LES of single jets in crossflow have been performed by Tyagi \& Acharya (2003), Iourokina \& Lele (2006), Peet (2006) and Renze, Meinke \& Schröder (2006), using periodic boundary conditions in the spanwise direction to mimic a row of cooling jets. Iourokina \& Lele (2006), Peet (2006) and Renze et al. (2006) have included the complete geometry (cooled channel, hole and plenum) used respectively by Pietrzyk, Bogard \& Crawford (1989) and Sinha, Bogard \& Crawford (1991) in the reference experiments. Note that only one row of holes is considered in these studies, which focus more on turbine blade FC than on combustion chamber wall cooling.

From the short literature overview presented above, the following comments can be made, regarding the understanding and modelling of FCFC:

(a) no detailed data about non-isothermal FCFC can be found in the open literature (apart from RANS results); only measurements of cooling efficiency and wall heat transfer are available. This is mainly because large temperature gradients impose small scale facilities for which achieving spatially resolved measurements is extremely challenging. A good way to proceed would be to rely on Direct Numerical or wall-resolved LargeEddy Simulations (Mendez \& Nicoud 2007). However, lack of experimental data remains problematic for obvious validation reasons,

(b) even in the isothermal case, only a few studies focus specifically on FCFC and for these particular studies, only injection side results have been reported. Moreover, only Miron (2005) used a supply channel on the suction side, with only part of the secondary flow passing through the holes. In Yavuzkurt et al. (1980a,b) and Gustafsson (2001), the fluid injected through the holes flows on the suction side in a plenum, with very small velocity. Detailed data relevant to the suction, aperture and injection regions is still lacking for FCFC, even in isothermal situation. Such data is needed to support the development of wall models able to represent the effects of effusion on both sides of the plate,

(c) a common difficulty in analysing experimental or numerical results relevant to FCFC is the dependency of the results on the position of the row where the flow is investigated. Several studies confirm the necessity to account for several rows before the FCFC situation is reached: Miron (2005) shows that the velocity profiles are still evolving after 10 rows of holes, while measurements have shown that cooling effectiveness stabilises only after a large number of rows (approximately 15 for Mayle \& Camarata 1975; Rouvreau 2001). Although 10-15 rows are often considered to be enough to study FCFC (Bazdidi-Tehrani \& Andrews 1994; Crawford et al. 1980; Metzger et al. 1973; Miron 2005), the independence of the results on the row position within the whole array of holes was never observed in previous studies and to the author's knowledge the existence of such 'self-similarity' is still an open question. This situation makes questionable the generality of the conclusions drawn from the investigation of spatially evolving boundary layers over multi-perforated plates. Moreover, it is in contradiction with the development of a wall model that is local in space and provides the fluxes through the effusion plate independently of the position on the perforated plate.

Due to the overall lack of relevant data, this is the authors' viewpoint that the isothermal case should be considered until more maturity about FCFC has been reached. Notably, gaining insight about the aspiration and aperture flow structure still remains to be achieved in the isothermal FCFC case. Moreover, obtaining data where the row number is not a parameter of the problem would introduce new perspectives in terms of FCFC modelling. It is the objective of this paper to address these issues. More precisely, a synthetic flow relevant to FCFC is computed by wall-resolved Large-Eddy Simulation. The configuration corresponds to a bi-periodic flow where both the aspiration and the injection sides of the plate are computed. Because periodicity is assumed in both direc- 


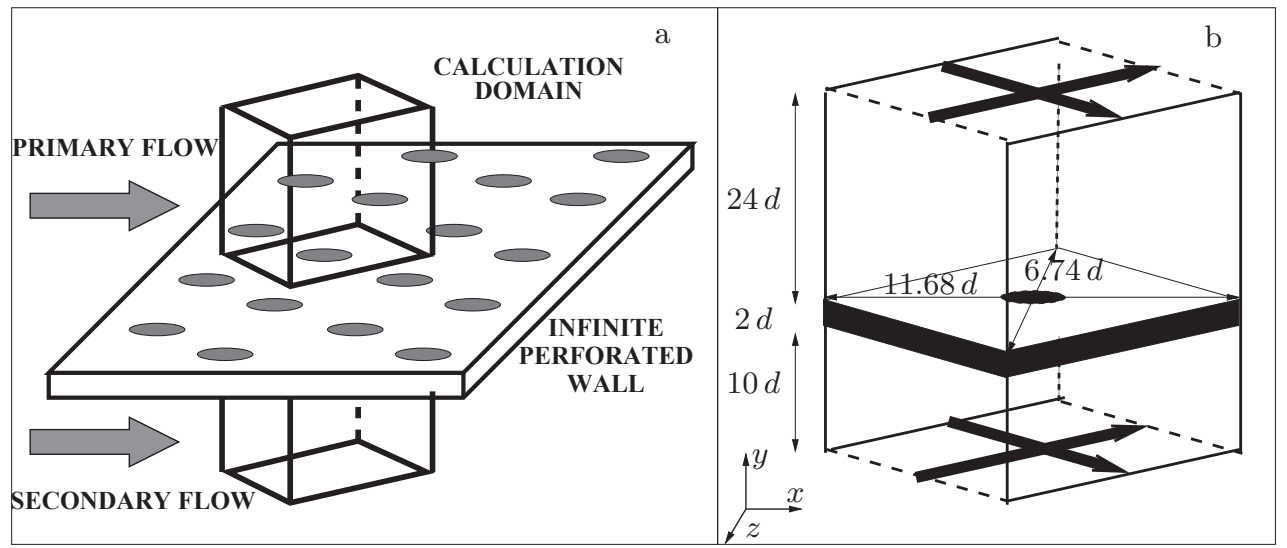

Figure 2. From the infinite plate to the 'bi-periodic' calculation domain. (a): Geometry of the infinite perforated wall. (b): Calculation domain centred on a perforation; the bold arrows correspond to the periodic directions. The dimensions of the computational domain are provided.

tions tangential to the plate, this flow corresponds to a case where the number of rows tends to infinity and the row position along the plate is no more a relevant parameter. The configuration of interest is described in $\S 2$ along with some numerical details about the LES code. Velocity profiles are presented in $\S 3$, where the similarities/differences between the classical situation with a finite number of rows and the present synthetic flow are highlighted. A detailed description of the flow features is provided in $\S 4$ and an analysis of the results in terms of wall modelling is presented in $\S 5$.

\section{Numerical method}

Results in the open literature show that the effusion flow highly depends on the configuration of interest: the flow at the end of a ten-row plate would be different from the one of a twenty-row plate. This situation is hardly tractable from a modelling point of view and not convenient for practical applications; the flow structure in three dimensional combustion chambers is neither simple nor known a priori, making the notion of number of upstream rows a fuzzy concept. Thus it has been decided to consider the asymptotic case where the flow is independent of the hole considered. The simulation is then designed to reproduce this 'fully-developed' turbulent flow with effusion. This choice presents several advantages:

(a) The computational domain may contain only a small number of perforations (for example one), with periodic boundary conditions to reproduce the whole geometry of an infinite plate, as it is suggested in figure 2. Due to the staggered arrangement of the perforations, the computational domain is diamond-shaped,

(b) The difficult question of the inlet and outlet boundary conditions in turbulent simulations (see Moin \& Mahesh 1998) is eluded,

(c) The computing effort is drastically reduced compared to spatially evolving flow, because the computational domain is smaller.

With such a periodic calculation domain, the objective is to have information about the structure of the flow far from the first rows, when the film is established. However, this periodic option raises a problem: natural mechanisms that drive the flow, such as pressure gradients in the periodic directions, are absent. The flow has to be generated artificially in a way that does not modify the details of the flow structure. 


\subsection{Generation of a periodic flow with effusion}

\subsubsection{Injection through the hole}

In experiments, channels are bounded by impermeable walls at the top and at the bottom. If used in conjunction with periodic boundary conditions in the tangential directions, this outer condition prevents the flow from reaching a statistically steady state with effusion, because the net mass flux through the perforation tends to eliminate the pressure drop between the cold and the hot domains. In the present simulations, characteristicbased freestream boundary conditions (Thompson 1990) are used at the top and bottom ends of the domain in order to impose the appropriate mean vertical flow rate. Note that Mendez, Nicoud \& Miron (2005) compared this boundary condition-based strategy to another, source terms-based, methodology and that the independence of the results on the method used to sustain the effusion was demonstrated.

\subsubsection{Primary and secondary flows}

For classical, periodic channel or pipe flows simulations, a volumetric source term $S_{(\rho U)}$ is added to the streamwise momentum conservation equation in order to mimic the effect of the mean streamwise pressure gradient that would exist in a non-periodic configuration. The source term is constant over space. For example, it can have the following form:

$$
S_{(\rho U)}=\frac{\left(\rho U_{\text {target }}-\rho U_{\text {mean }}\right)}{\tau}
$$

The source term compares a target momentum value, $\rho U_{\text {target }}$, with the spatial-averaged momentum in the channel, $\rho U_{\text {mean }}$. The time scale $\tau$ characterises the relaxation of $\rho U_{\text {mean }}$ towards its target value. This approach can be generalised to the case of an effusion configuration, making use of a source term of the previous form in each channel to generate the primary and the secondary flows and no source term within the hole. Note however that no source term is required in the aspiration side since the target velocity for the secondary flow can be imposed through the boundary condition at the bottom end of the domain; it is then convected throughout the bottom channel without the need of extra external forcing.

\subsection{Numerical simulations}

The smallest domain that can reproduce the geometry of an infinite plate with staggered perforations contains only one hole and is diamond-shaped (see figure 2). In the present isothermal simulations, the computational domain is divided into two channels. The upper one, denoted by ' 1 ', represents the combustion chamber, with a primary flow of 'hot' gases. The second one, denoted by ' 2 ', represents the casing, with a secondary flow of 'cooling' air. The height of the channels are $h_{1}=24 d$ and $h_{2}=10 d$ respectively, where $d=5 \mathrm{~mm}$ is the diameter of the cylindrical aperture. The upper and lower limits of the domain are far enough from the zone of interest to avoid any spurious effect of boundary conditions on the flow near the perforated plate. The channels are separated by a perforated plate of thickness $10 \mathrm{~mm}$, the aperture being angled at $\alpha_{g}=30^{\circ}$ with the plate, in the streamwise direction, without any spanwise orientation. The thickness of the plate being $10 \mathrm{~mm}$ and holes being angled at $30^{\circ}$ with the plate, the hole lengthto-diameter ratio is 4 . The diagonals of the computational domain are $z=0$ and $x=0$ and their lengths equal the hole-to-hole distance, viz. $11.68 d$ in the streamwise direction $(z=0)$ and $6.74 d$ in the spanwise direction $(x=0)$. The centre of the hole is located at $x=0, y=0, z=0$ for the injection side (hole outlet) and at $x=-3.46 d, y=-2 d$, $z=0$ for the suction side (hole inlet). 


$\begin{array}{ccccc}\text { Name } & \text { Number of Cells } & \text { Hole points } & \text { Hole size } & \boldsymbol{\Delta}^{+} \\ \text {COARSE } & 150,000 & 5 & 1 \mathrm{~mm} & 20 \\ \text { MEDIUM } & 1,500,000 & 15 & 0.3 \mathrm{~mm} & 5 \\ \text { FINE } & 25,000,000 & 45 & 0.1 \mathrm{~mm} & 2\end{array}$

TABle 3. Main characteristics of the three grids considered. Column 2: number of tetrahedral cells, Column 3: typical number of points along the hole diameter, Column 4: typical cell size within the hole, Column 5: average cell size near the wall in wall units (injection side) in the three directions (tetrahedra are isotropic).

The characteristics of the three grids used to represent the flow domain are reported in table 3. In all cases, the mesh contains essentially isotropic tetrahedral cells, without particular direction of stretching. In particular, average grid spacing is equal in the three directions: $\Delta x^{+}, \Delta y^{+}$and $\Delta z^{+}$at the wall are represented by a unique value noted $\Delta^{+}$ in table 3. Since the expected flow structure is 3D and complex, the size of the cells is kept roughly constant within the aperture and wall regions $(-2 d<y<2 d)$ so that the numerical errors are kept to their minimum is the region of interest. Further from the solid wall, the mesh is stretched in order to minimise the total number of degrees of freedom, the cell-to-cell volume ratio being always less than 1.02.

The main characteristics of the simulations discussed in this paper are gathered in table 4. Runs A, B and C correspond to the 1-hole configuration depicted in figure 2 (right) and discretised respectively by the COARSE, MEDIUM and FINE meshes described in table 3. By making use of a bi-periodic computational domain containing only one hole, one enforces the hole-to-hole distance to play a major role in the simulation. Any turbulence length scale greater than half the domain size would not have enough room to appear and, more importantly, jet-to-jet interaction cannot take place. The aim of Run D, which corresponds to a 4-hole computational domain obtained by duplicating the MEDIUM mesh twice in each tangential direction, is to assess how the results are modified, if they are, by the choice of a 1-hole bi-periodic domain. In order to save CPU time, the initial condition for this 4-hole computation (Run D) is a four times duplicated version of an established solution from the 1-hole simulation (Run B).

The values of discharge coefficient $C_{D}$ and blowing ratio are reported in table 4 . The discharge coefficient $C_{D}$ is related to the pressure difference $\Delta P$ between the secondary and primary channels. It is $C_{D}=\sqrt{\rho_{j} V_{j}^{2} / 2 \Delta P}$, where $V_{j}$ is the bulk velocity in the jet and $\rho_{j}=1.13 \mathrm{~kg} \mathrm{~m}^{-3}$ is the mass density in the jet. Note that variations of $\rho_{j}$ in the calculation are small due to the isothermal configuration. Two ways of calculating the blowing ratio are reported: $M$ is the ratio between the velocity in the jet core (measured 3 diameters downstream of the hole centre) and the velocity at the centre of the primary channel while $M_{b}$ is the ratio between the bulk velocity in the hole and the bulk velocity in the region where the jet and the main flow interact $(0<y<6 d)$, viz. $U_{1} \approx 5.0 \mathrm{~m} \mathrm{~s}^{-1}$. These two numbers convey essentially the same information, $M_{b}$ being more precisely defined and less sensitive to local changes; $M$ was added to facilitate the comparison with the experimental data where the bulk velocities are not available. The bulk velocity in the 'hot' stream is close to $U_{2} \approx 2.2 \mathrm{~m} \mathrm{~s}^{-1}$.

Based on the bulk velocity in the 'hot' stream and the streamwise distance between two holes, the flow through time is $F T T=0.0117 \mathrm{~s}$. All the statistics presented in this paper have been accumulated over 23 FTT. Regarding Run D, the statistics have been accumulated from the $32^{\text {nd }}$ FTT after the initialisation of Run D from a snapshot of Run 


\begin{tabular}{cccccccc}
\hline Run & Domain & Grid & $\mathbf{C}_{\mathbf{D}}$ & $\mathbf{V}_{\mathbf{j}}\left(\mathrm{m} \mathrm{s}^{-1}\right)$ & $\mathbf{\Delta} \mathbf{P}(\mathrm{Pa})$ & $\mathbf{M}$ & $\mathbf{M}_{\mathbf{b}}$ \\
$\mathbf{A}$ & & & & & & & \\
1-hole & COARSE & 0.56 & 4.85 & 43 & 1.53 & 1.07 \\
$\mathbf{C}$ & 1-hole & MEDIUM & 0.67 & 5.67 & 41 & 1.58 & 1.12 \\
$\mathbf{D}$ & 1-hole & FINE & 0.69 & 5.84 & 41 & 1.58 & 1.17 \\
& & MEDIUM & 0.67 & 5.67 & 41 & 1.58 & 1.12
\end{tabular}

TABLE 4. Main characteristics of the presented simulations.

B. It is assumed that any jet-to-jet interaction would have had enough time to appear during the total of 55 FTT that have been computed. Since a detailed analysis of the snapshots over the simulation showed no such event, it is believed that the micro-jets are not subjected to collective interaction, at least for the operating point considered in this study. Note that unless otherwise stated, the flow through time, the bulk velocity in the hole $V_{j}$ obtained in Run $\mathrm{C}\left(V_{j}=5.84 \mathrm{~m} \mathrm{~s}^{-1}\right)$ and the diameter of the hole are the time, velocity and length scales.

All simulations are carried out with the LES code AVBP developed at CERFACS (www.cerfacs.fr/cfd/avbp_code.php). It is based on a fully explicit cell-vertex formulation and solves the compressible Navier-Stokes equations on unstructured meshes for the conservative variables (mass density, momentum and total energy). AVBP is dedicated to LES and has been widely used and validated in the past years in all kinds of configurations (Moureau et al. 2005; Schmitt et al. 2007; Schönfeld \& Rudgyard 1999), and notably in jet-in-crossflow cases (Prière et al. 2005, 2004). The present simulations are based on the WALE sub-grid scale model (Nicoud \& Ducros 1999), which provides the appropriate damping of the sub-grid scale viscosity in the solid walls region. The numerical scheme is the TTGC scheme (Colin \& Rudgyard 2000): this essentially non dissipative scheme was specifically developed to handle unsteady turbulent flows with unstructured meshes. It is third order accurate in both space and time. The solid wall that represents the perforated liner is an adiabatic non-slipping wall. The boundary conditions at the lower and upper limits of the domain are characteristic-based freestream conditions. In the simulations, the desired conditions are obtained by imposing the streamwise bulk velocities (spatial and time-averaged velocities) in the two channels thanks to constant source terms and the pressure drop thanks to the upper and lower boundary conditions. The relaxation time value for the source term (see Eq. 2.1) is approximately $\tau=10 \Delta t$ for all the computations, where $\Delta t$ is the explicit time step of the time integration as given by the CFL stability criterion (CFL is fixed to 0.7 to ensure the stability of the numerical scheme).

The geometrical and flow characteristics described above have been chosen to allow a comparison with the isothermal experimental data of Miron (2005). The so-called LARA experiment consists in two channels of height $h=120 \mathrm{~mm}$ and width $l=400 \mathrm{~mm}$ separated by a plate perforated with twelve rows of holes of diameter $5 \mathrm{~mm}(0.5 \mathrm{~mm}$ is a typical value for gas turbines). The operating point considered in this paper is such that the pressure drop across the plate and blowing ratio are $\Delta P=42 \mathrm{~Pa}$ and $M=1.54$ respectively. The section containing the perforated plate is preceded by two long ducts: when reaching the test section, the flows correspond to fully developed channel flows in each duct. The Reynolds number for the primary 'hot' flow (based on the duct centreline velocity and the half height of the rectangular duct) is $R e_{1}=17750$, while it is $R e_{2}=8900$ for the secondary 'cold' flow. The Reynolds number in the hole, based on the 


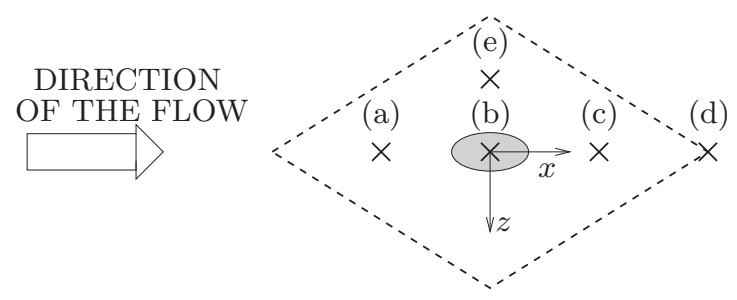

\begin{tabular}{|c|c|c|}
\hline & $x / d$ & $z / d$ \\
(a) & -2.92 & 0 \\
(b) & 0 & 0 \\
(c) & 2.92 & 0 \\
(d) & 5.84 & 0 \\
(e) & 0 & -1.5 \\
\hline
\end{tabular}

Figure 3. Projected location of the profiles displayed in the paper: (a), (b), (c), (d) and (e). The streamwise $(x / d)$ and spanwise $(z / d)$ locations of the points are reported in the table. Profiles are measured from the wall $(y / d=0)$ to the centre of channel $1(y / d=12)$. The dashed line represents the projection of the calculation domain.

momentum in the jet core and the hole diameter is $R e_{h}=2600$. Further details about this experiment can be found in Miron, Bérat \& Sabelnikov (2004) and Miron (2005).

\section{First-order statistics}

In this section, low order statistics from Runs A, B, C and D are analysed in order to establish the numerical accuracy of the numerical data. Comparisons with the experimental measurements of Miron (2005) are provided when possible. The ninth row of holes has been chosen for this purpose because it is the location where measurements are most numerous. Note however that the experimental results depend on the row considered so that a perfect agreement cannot be expected with the numerical results for which the row position within the plate is not a relevant parameter. Establishing the numerical accuracy of the LES data by comparing runs A-D is thus a necessary step. Once the numerical accuracy is established, the numerical/experimental comparisons can serve as a mean to investigate the differences between a spatially evolving and a homogeneous turbulent flow with effusion.

\subsection{One-point statistics}

The five locations where statistics profiles will be displayed are shown in figure 3. Positions (a)-(d) belong to the mid-plane of the computational domain and are aligned with the computed jet, either upstream or downstream; (e) is located $1.5 d$ apart from the jet exit.

Three levels of grid are compared in figures 4 and 5. Overall, the differences between Runs B and C (MEDIUM-FINE) are very small compared to the differences between Runs A and B (COARSE-MEDIUM). As an illustration, the overall difference in the streamwise velocity is $0.065 V_{j}$ between Runs A and B while it is only $0.017 V_{j}$ between $\mathrm{B}$ and $\mathrm{C}$. In figure 5 , the level of vertical velocity and velocity fluctuations is clearly smaller for Run A while Runs B and C are very similar (the experimental results will be discussed later in section 3.3). Although the concept of grid convergence is not clearly defined for LES without explicit filtering, these results indicate that the MEDIUM grid is enough to reproduce the main features of the flow considered, the FINE mesh providing the most detailed results. From figure $4(a)$, the flow upstream of the hole is affected by the presence of the cooling film formed by the former jets, at least for $y \leq 6 d$. The interaction between this incident flow and the jet is observed in figure $4(b)$. High values of velocity are observed at the outlet of the hole (near $y=0)$. The jet strongly 

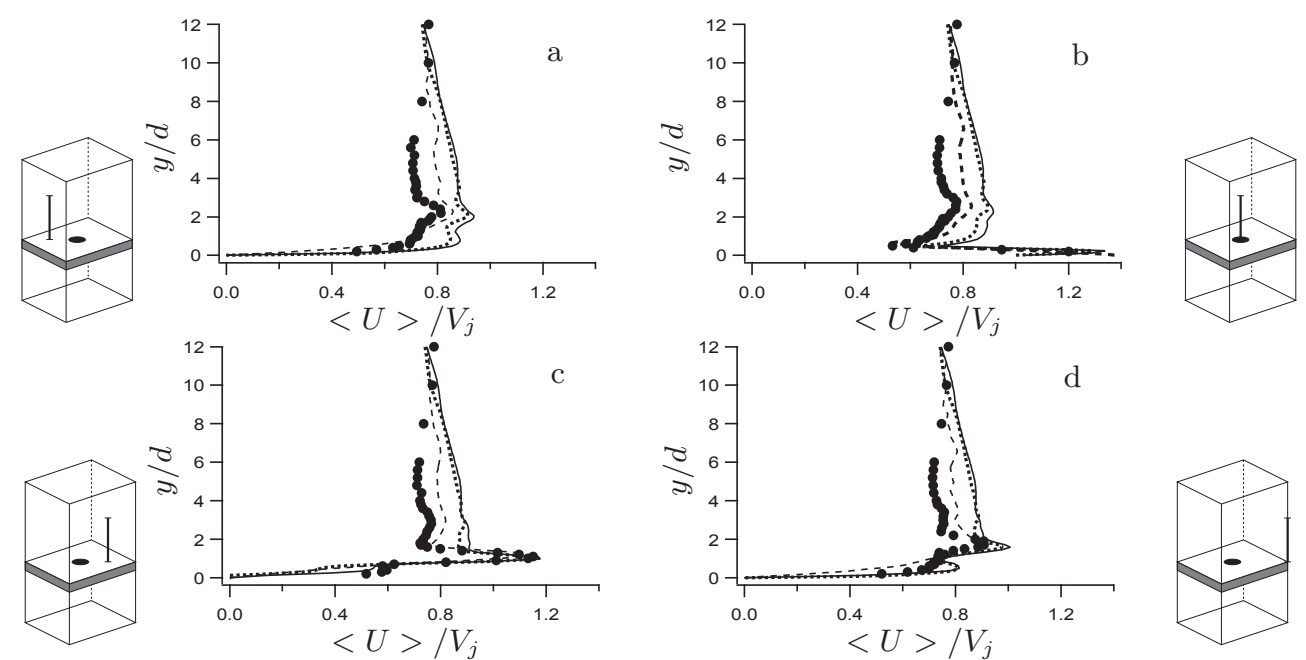

FiguRE 4. Time-averaged streamwise velocity profiles at four locations: comparison between experimental results $(\bullet)$, Run A ( ---$)$, Run B ( - ) and Run C ( ….... Graphs correspond to the locations (a), (b), (c) and (d) presented in figure 9.

modifies the streamwise velocity profile downstream of the hole, as shown in figure $4(c)$. The characteristic form of effusion profiles is observed, with a peak $(y / d \approx 1)$ marking the jet just upstream, and a second peak that represents the film created by injection through the former holes $(y / d \approx 3)$. This second peak is more pronounced in the coarse grid results (Run A). Further downstream (figure 4d), the jet looses its strength and progressively mixes with the film. Also, the velocity near the wall increases compared to figure 4(c); this is an effect of the entrainment process (see for example Yavuzkurt et al. 1980a): when the blowing ratio is high enough (typically greater than 0.5), the jets separate and penetrate deeply in the boundary layer. The main flow bypasses the jet and experiments a movement towards the plate, resulting in negative values of wallnormal velocity around the jet (see $\S 4.4$ and 4.5). An effect of the entrainment process is to reattach the main flow downstream of the jet, inducing the increase in streamwise velocity observed in figure $4(d)$.

Regarding the potential effect of the number of holes included in the bi-periodic domain, it is important to note that the 1-hole (Run B) and 4-hole (Run D) configurations lead to very similar results. This is illustrated in figures 6 and 7 where the profiles of the averaged and root-mean-square (RMS) streamwise and normal velocity components are shown for two locations: one downstream of the hole (position (d) in figure 3), and the second one on the side of the hole (position (e) in figure 3). In these plots the profiles corresponding to the four holes of the 4-hole computation are represented by the same line type (solid) since there is no statistical difference between these profiles. The differences observed are due to the lack of statistical convergence and provide an easy way to estimate the statistical uncertainty in the plotted profiles. Given this error bound, there is no difference between the 1-hole and the 4-hole computations. The same conclusion was drawn for all the one point statistics comparisons performed between the two configurations. Note in figure $7(b)$ the negative normal velocity in the region $0<y<2 d$ and large streamwise velocity very close to the wall in figure $7(a)$. These features result from the bypass by the main stream of the two jets upstream position (e) and located at $(x=-5.84 \mathrm{~d}, z=0)$ and $(x=-2.92 \mathrm{~d}, z=-3 \mathrm{~d})$. Regarding the RMS of velocity at position (e), there is a peak very close to the wall for the streamwise component, while 

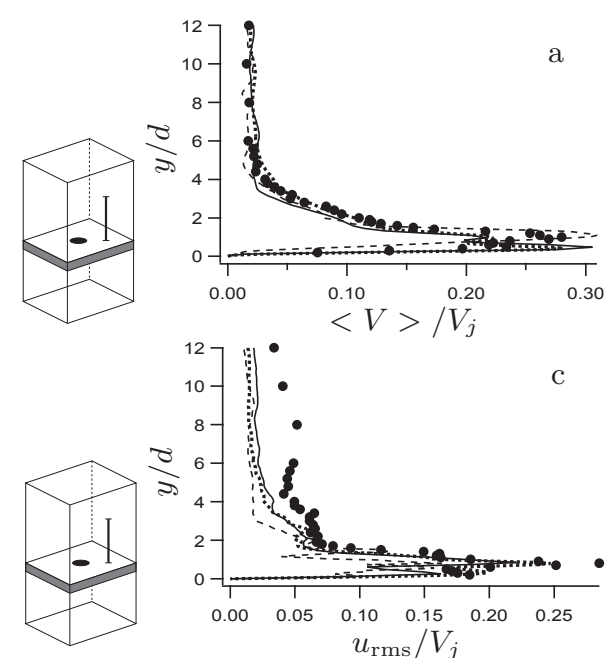

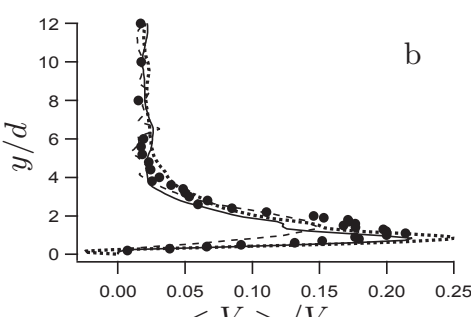

$<V>/ V_{j}$
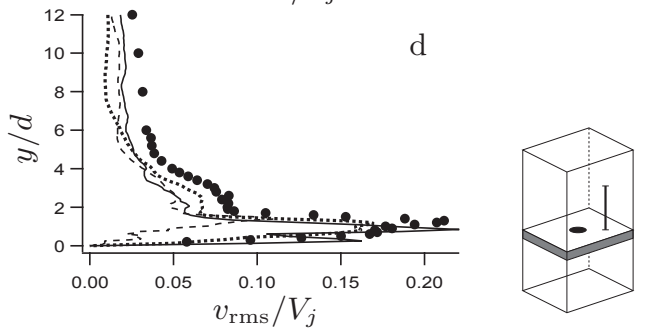

FiguRE 5. Velocity profiles: comparison between experimental results ( • ), Run A ( --- ), Run $\mathrm{B}(-)$ and Run C ( …....). (a): time-averaged vertical velocity at station (c), $x=2.92 d$, (b): time-averaged vertical velocity at station (d), $x=5.84 d$, (c): streamwise RMS velocity at station (c), $x=2.92 d,($ d): vertical RMS velocity at station (c), $x=2.92 d$.

the maximum of the normal fluctuations is as far as 3 diameters away from the wall, with a secondary peak very close to the wall as well. Using the local friction velocity as a velocity scale, the peak of streamwise RMS is located at 13 wall units from the solid boundary and its value is close to 3.2. This suggests that at location (e) where the effects of upstream jets are not felt directly, the classical wall scaling holds reasonably and the classical wall turbulence structure tends to be recovered. Note however that the value of the secondary peak in normal RMS corresponds to $0.012 V_{j}$ or 0.25 wall units, a value smaller than the classical value close to unity in wall bounded turbulent flows. One reason could be that the redistribution process via the velocity pressure fluctuations does not have time enough to operate. In any case, the flow structure in position (e) is closer to the classical solid wall situation than position (d) where the mean and RMS profiles are dominated by the jet. Indeed, from figure $6(c, d)$, the location of maximum velocity fluctuations is roughly 1.3 diameter above the plate where $u_{\mathrm{rms}}$ and $v_{\mathrm{rms}}$ share the same value, viz. $0.16 V_{j}$; in local wall units, this corresponds to 3.5 for the peak value and 110 for its distance to the wall, very far from the classical values for attached turbulent flows (except for the peak value of $u_{\mathrm{rms}}$ ).

\subsection{Two-point correlations}

Typical streamwise autocorrelation coefficients for the streamwise and normal velocity components $\left(C_{\mathrm{uu}}\right.$ and $\left.C_{\mathrm{vv}}\right)$ are depicted in figure 8 . These profiles were obtained by postprocessing 50 independent solutions of the 4-hole run and 104 1-hole run snapshots. The four hole regions in the 4-hole run were subsequently averaged together to obtain the presented results. In the centre of figure 8 , the reference points location for the computation of the streamwise two-point correlations is also depicted. In one case (figure $8 a, c$ ) the reference point (which corresponds to zero streamwise distance in the figure) is located above a hole and the end point is located above the next hole in the downstream direction. In the other case (figure $8 b, d$ ), the reference point is located at half the distance between two consecutive lines of holes. The lines over which the correlations were computed are located $1.2 d$ above the injection plate. The streamwise hole-to-hole dis- 

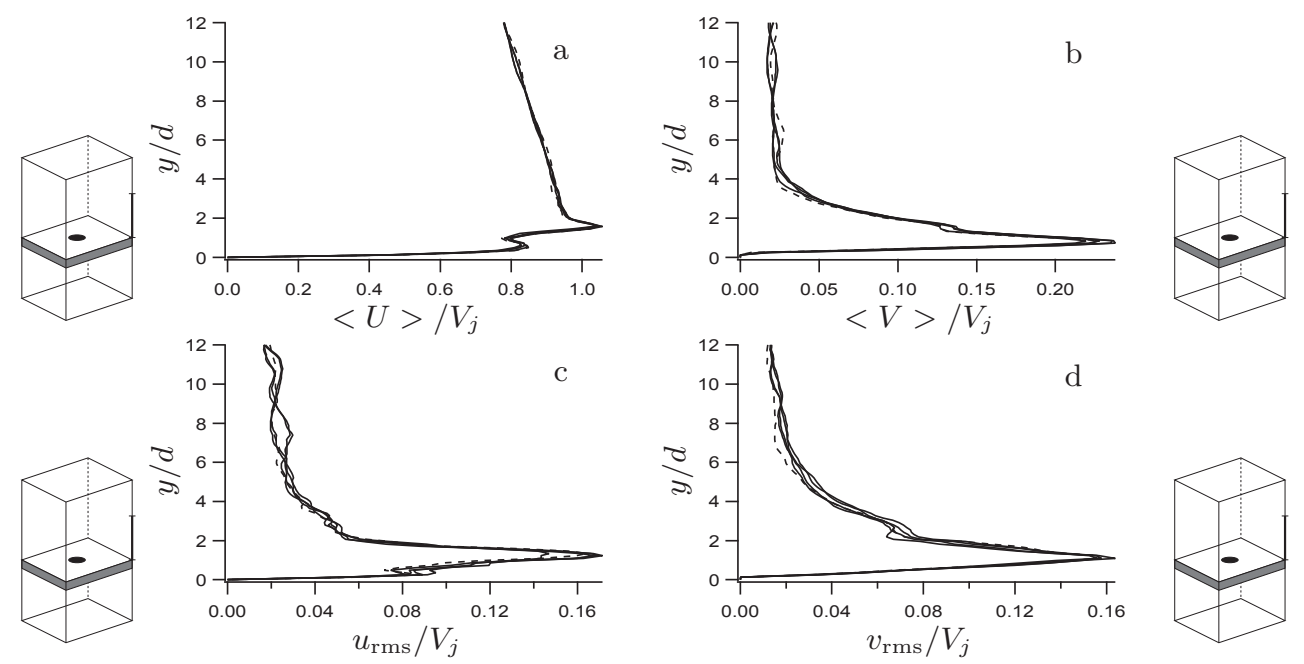

Figure 6. Velocity profiles from the 1-hole ( --- , Run B) and the 4-hole ( - , Run D) computations at position (d) in figure 9 (viz. in between two consecutive holes in the spanwise or streamwise direction): (a): time averaged streamwise velocity, (b): time averaged normal velocity, (c): RMS of streamwise velocity, (d): RMS of normal velocity.
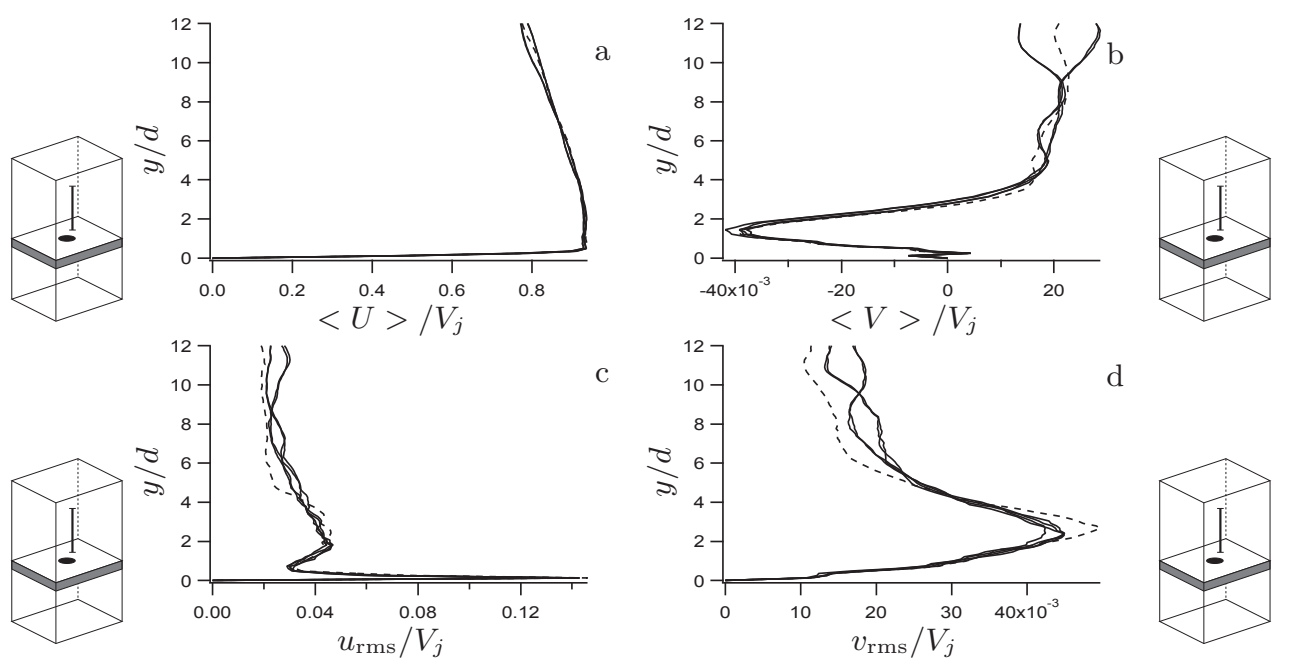

Figure 7. Velocity profiles from the 1-hole $(---$, Run B) and the 4-hole ( - - , Run D) computations at position (e) in figure $9(x=0, z=-1.5 d)$ : (a): time averaged streamwise velocity, (b): time averaged normal velocity, (c): RMS of streamwise velocity, (d): RMS of normal velocity.

tance, $11.68 d$, is used to make the streamwise distance dimensionless. All graphs show a decrease of the autocorrelation coefficients, which reach small values before half the streamwise hole-to-hole distance. Note that the effect of the periodic boundary conditions clearly appears in the 1-hole case with values of autocorrelation coefficients going to 1.0 at a scaled streamwise distance of 1.0. This behaviour is not observed for the 4hole results where the points located one streamwise hole-to-hole distance apart are not correlated. Figures $8(a, c)$ suggest that in Run D, with 4 holes computed, no jet-to-jet interaction occurs, supporting the idea that the use of a periodic domain containing one aperture does not break any natural interaction. In general, with the exception of the 

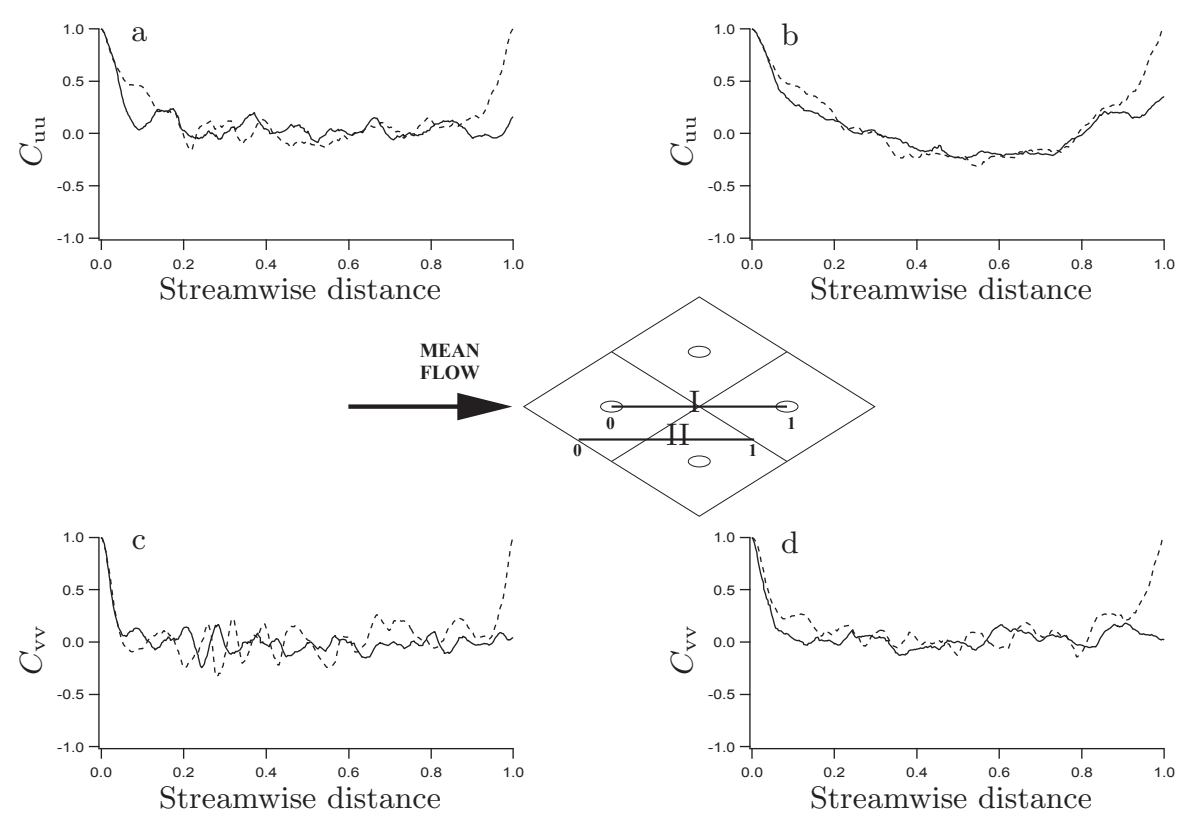

FIgURE 8. Streamwise autocorrelation coefficients for the streamwise [plots (a)\&(b)] and normal [plots (c)\&(d)] velocity components from the 1-hole ( --- , Run B) and the 4-hole ( - , Run D) computations at 1.2 diameter above the liner for paths I [plots (a)\&(c)] and II [plots (b)\&(d)]. The sketch in between the plots depicts the paths along which the correlations have been computed.

periodicity effect, no major difference appears between the 1-hole and the 4-hole runs. Note however that non-negligible differences between the 1-hole and the 4-hole runs are sometimes visible (for example in figure $8(a)$ at a reduced separation of 0.1). Given the very good agreement observed previously for the time-averaged and RMS of streamwise and normal velocity components (figures 6 and 7), these differences are most likely due to a lack of statistical convergence. From a physical point of view, figure 8 also suggests that the micro-jets have a strong effect on the turbulence structure. Turbulent integral length scales $L_{\mathrm{uu}}$ and $L_{\mathrm{vv}}$ can be assessed by integrating $C_{\mathrm{uu}}$ and $C_{\mathrm{vv}}$ from 0 (reference point) to 0.5 (half the hole-to-hole streamwise distance): this leads to $L_{\mathrm{uu}}=0.7 d$ and $L_{\mathrm{vv}}=0.35 d$ for line I and $L_{\mathrm{uu}}=1.1 d$ and $L_{\mathrm{vv}}=0.7 d$ for line II. Two conclusions can be drawn from these assessments: (a) the turbulent integral length scales $L_{\mathrm{uu}}$ and $L_{\mathrm{vv}}$ are always of order $d$ and not of order of the hole-to-hole distance, (b) the turbulent integral length scales are significantly (30-50\%) smaller along lines crossing the micro-jets and larger otherwise. Although not displayed, spanwise autocorrelation coefficients have been calculated too. No major difference between the 1-hole and 4-hole results could be observed, the agreement being actually better than for the streamwise two-points correlations presented in figure 8.

Single-hole computations with periodic boundary conditions allow to account for the effect of the jets contained in the neighborhood of the jet considered: it is known that jet interaction can considerably modify the jets behaviour (see for example Yu, Ali \& Lee 2006). However, long-distance interactions, such as acoustic interactions (as in Staffelbach, Gicquel \& Poinsot (2006), with flames exciting each other in a periodic simulations of a gas turbine combustion chamber) cannot be reproduced. In the present paper, such type of collective interactions has not been observed in the 4-hole computation, supporting the idea that the 1-hole computation performed with the finest grid (Run C) indeed 
contains all the physics relevant to the turbulent flow over the infinite perforated plate considered. Note however that this conclusion cannot be true for all the geometries. Figure $8(b)$ shows for example that if the hole streamwise spacing was twice smaller, $C_{\mathrm{uu}}$ would clearly be controlled by periodicity. It is difficult to state for which conditions jet-to-jet interactions may occur, but small row-to-row spacing (typically of order 3-4d) would probably necessitate computations including a larger number of holes.

\subsection{Spatially evolving versus homogeneous flow}

From the previous sections, one can conclude that the computational domain and spatial resolution are appropriate for the flow of interest. Note also that the same flow configuration has been computed with another LES code (Ham \& Iaccarino 2004), called CDP and developed at the Center for Turbulence Research (Stanford University, California). The detailed comparison is presented in Mendez et al. (2006). The very good agreement between the two codes strongly supports the idea that numerical or sub-grid scale modelling errors have no significant effects on the results presented in this paper. It is then justified to consider Run $\mathrm{C}$ as a reference solution relevant to a bi-periodic (fullydeveloped) turbulent flow with effusion. The next natural question to address regards the similarities/differences of this flow compared to the more classical spatially evolving configuration where the position within the array of holes is a relevant parameter.

In the LARA experiment, Miron (2005) investigated the flow within two parallel channels separated by a 12-rows perforated plate. The experimental data base provides velocity profiles in the streamwise and vertical directions on the injection side of the plate, at row 9. Comparisons are made with experimental profiles at locations (a), (b), (c) and (d) of figure 3 and recalled in figure 9. Note that no measurement is available at position (e). From figures 4 and 5, a general good agreement is obtained between the simulations and the experiment. Surprisingly, Run A (COARSE grid) seems to better reproduce the experimental data than Runs B and C (finer grids). However, the near-wall region is not discretised finely enough: this leads to important errors on the velocity gradient at the wall (figure $4 d$ ) as well as a significant under-estimation of the velocity fluctuations (figure $5 c, d$ ). These errors are also related to an insufficient description of the vortical structure of the flow, the entrainment process being not correctly reproduced with the coarsest grid.

Regarding Runs B and C (MEDIUM and FINE mesh), the behaviour of the streamwise velocity in the near wall region is well represented: the velocity peak due to the jet is located as in the experiment (figure $4 c$ ) and the RMS peak is well reproduced (figure $5 c$ ) in both shape and level. At the same time, significant differences can be found in the film core region $(y>2 d)$ where the numerical streamwise mean velocity is systematically larger than the experimental values. A closer investigation of the experimental data base supports the idea that this difference is mainly due to the difference between the configurations that are studied: recall that simulations characterise the flow around an infinite perforated plate while measurements correspond to the ninth row of a spatially evolving flow. From figure 10, which displays the mean streamwise velocity profiles at several locations along the perforated plate, the velocity of the film core (above the jet) tends to increase with the number of upstream rows. Measurements are performed 3 diameters downstream of the centre of the hole located in the middle of each row, far from the lateral walls (see figure $10 a$ ). Velocity profiles show the formation of a film created by effusion through the plate. The jets interact together to form a film that develops in the 'hot' side, modifying the primary flow in the neighborhood of the plate. Time-averaged streamwise velocity profiles (figure 10b) are characterised by three peaks: the first one $\left(\mathrm{P}_{\mathrm{a}}\right)$, next to the wall, represents the jet core $(y / d \approx 1)$. The second peak 


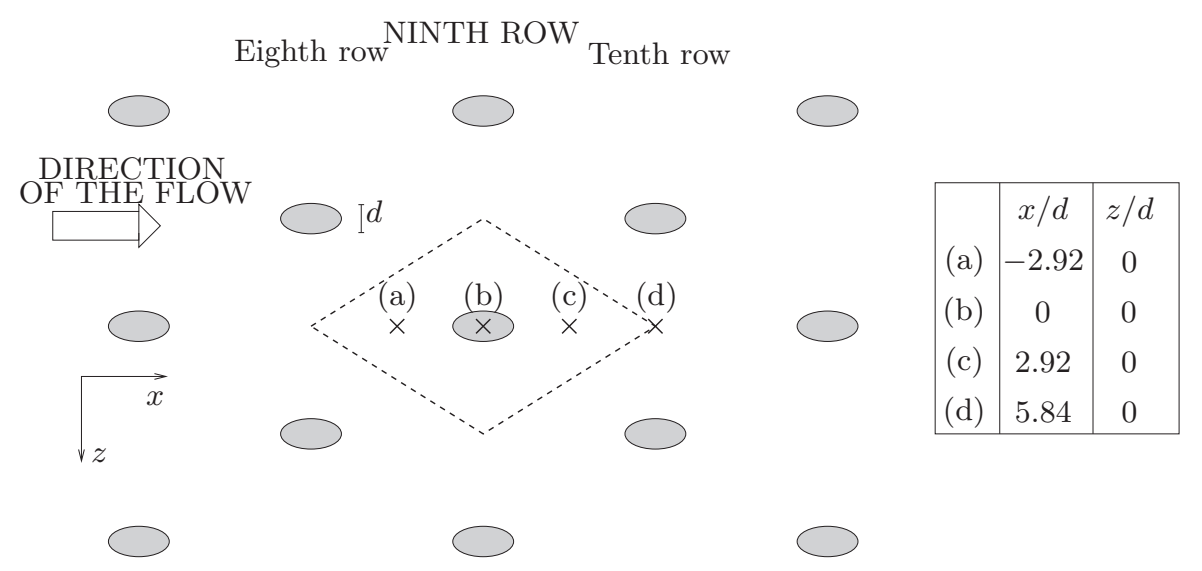

Figure 9. Zoom on the ninth row of the experimental test rig. Projected location of the profiles measured in the experiment: (a), (b), (c) and (d). The streamwise $(x / d)$ and spanwise $(z / d)$ locations of the points are reported in the table. Profiles are measured from the wall $(y / d=0)$ to the centre of channel $1(y / d=12)$. The projection of the computational domain $(---)$ is also represented.
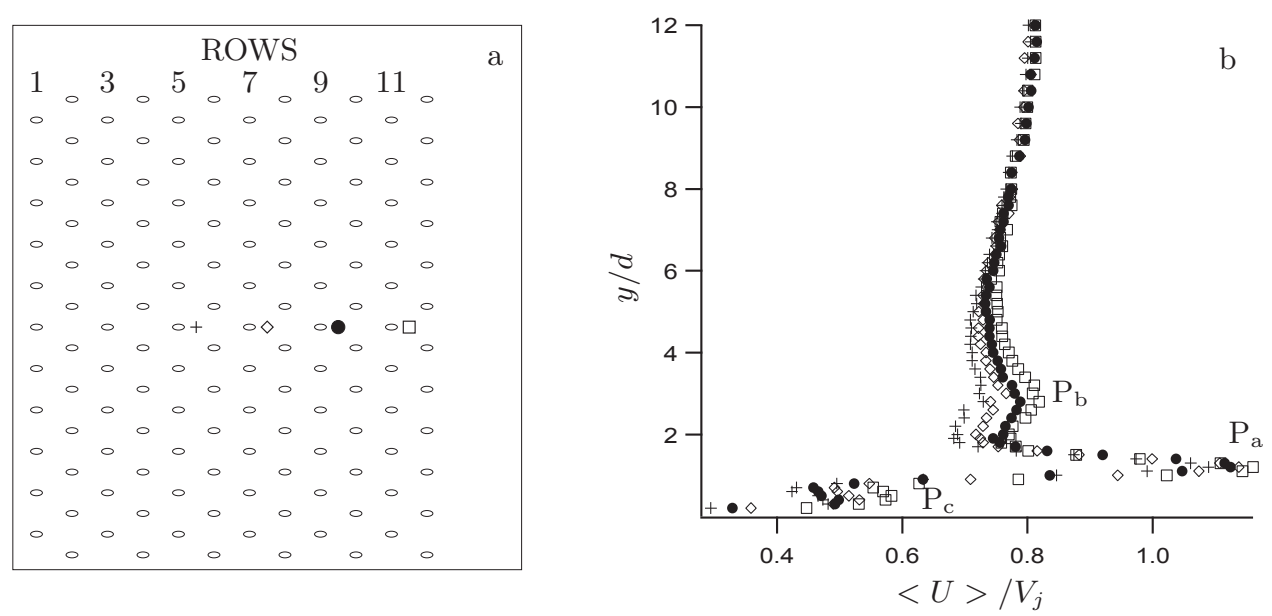

Figure 10. Experimental measurements: (a): perforated zone of the LARA plate with the measurements locations $(+, \diamond, \bullet, \square),($ b): time-averaged streamwise velocity profiles evolution in the injection region: + : fifth row, $\diamond:$ seventh row, $\bullet:$ ninth row, $\square:$ eleventh row. Profiles are measured 3 diameters downstream of each row, on the centreline plane.

$(y / d \approx 3)$ represents the film core $\left(\mathrm{P}_{\mathrm{b}}\right)$, which results from the interaction of all the upstream jets with the main flow. The presence of a secondary velocity peak, located below the jet core and due to the entrainment process, can also be observed $\left(\mathrm{P}_{\mathrm{c}}\right)$ at $y / d \approx 0.5$.

An important feature visible from figure 10 is that the peaks behave differently: the peak related to the jet just upstream does not change a lot from one row to the other, whereas the peaks corresponding to the film core and the entrainment process are highly influenced by the number of rows upstream of the measurement location. Since $\mathrm{P}_{\mathrm{a}}$ is related to the jet just upstream and the flow rate is roughly uniform along the plate, this peak does not depend on the position over the plate. On the contrary, $\mathrm{P}_{\mathrm{b}}$ and $\mathrm{P}_{\mathrm{c}}$ are directly related to the velocity of the main flow just upstream the hole and thus their 
amplitude depend on the number of row upstream, viz. on the position over the plate. It is also obvious from figure 10 that the experimental results are not established at row 9: the jet core is rapidly established, but peaks $\mathrm{P}_{\mathrm{b}}$ and $\mathrm{P}_{\mathrm{c}}$ continue to evolve. Now, since in the computation these peaks have reached an established state because of the enforced periodicity in the streamwise direction, a significant difference is found between the two data sets (figure $4 \mathrm{c}, \mathrm{d}$ ), the streamwise velocity near peaks $\mathrm{P}_{\mathrm{b}}$ and $\mathrm{P}_{\mathrm{c}}$ being larger in the bi-periodic case. Note that this accumulation effect is not reproduced in the coarse grid simulation, as the accumulation of fluid near the wall is a consequence of the entrainment process. This explains the fortuitous better agreement observed for the coarse resolution (Run A) in the film core region (figure 4).

Although differences in the mean streamwise velocity appear between the synthetic flow and the spatially evolving situation, it is fair to believe that conclusions drawn from the simulations do not apply only to 'infinite' configurations. Even if the film still evolves after 11 rows (figure 10), the peak marking the jet upstream is established very quickly, after only 5 rows. It is thus expected that the momentum flux induced by the jet does not change much as a function of the row number. The viscous fluxes at the wall probably need a larger number of rows to be established, but their contribution is small (see §5) compared to the inviscid term for blowing ratio greater than unity (this conclusion does not hold for cases with blowing ratio much smaller than unity but they are not representative of practical FCFC applications). Thus it is expected that any model built from the present numerical data would be useful even for FCFC plates with a moderate number of rows (5-10 say).

At last, for quantities that are not directly affected by the accumulation effect due to the periodicity enforced in the streamwise direction, comparisons between the two configurations show a very good agreement for the fine grids, as shown for the timeaveraged vertical velocity (top of figure 5) for stations (c) and (d) or for the RMS velocities (bottom of figure 5).

\section{Flow structure}

The complete flow structure is detailed in this section, focusing on all the three regions that compose the effusion cooling configuration: suction side, aperture and injection side. The vortical structure of the flow, which is a topic of particular interest in jets in crossflow is also described. Results from the FINE grid (Run C) have been used in this section since they contain more small scale information and provide the most complete flow description.

\subsection{General flow description}

Figure 11 presents contours and isolines of the time-averaged pressure and velocity magnitude from Run $\mathrm{C}$ on the mid plane $(z=0)$ : the pressure in the lower channel $P_{2}$ is higher than that of the upper channel, $P_{1}$, leading to injection of fluid into the upper channel. Effusion cooling is then a suction process for the lower channel and an injection process for the upper channel.

Several general features of the flow can be observed in figure 11: the pressure difference across the plate is essentially due to strong variations at the entrance of the hole (1). In this zone, the variations of pressure are as large as the total pressure drop. The value of pressure on the injection side $P_{1}$ is almost reached just after the entrance of the hole, inducing a strong acceleration of the fluid. Due to the sharp edge at the entrance of the hole, the jet separates (2). Walters \& Leylek (2000) also obtained this flow organisation in their RANS calculations for similar configurations. They define two regions in the 

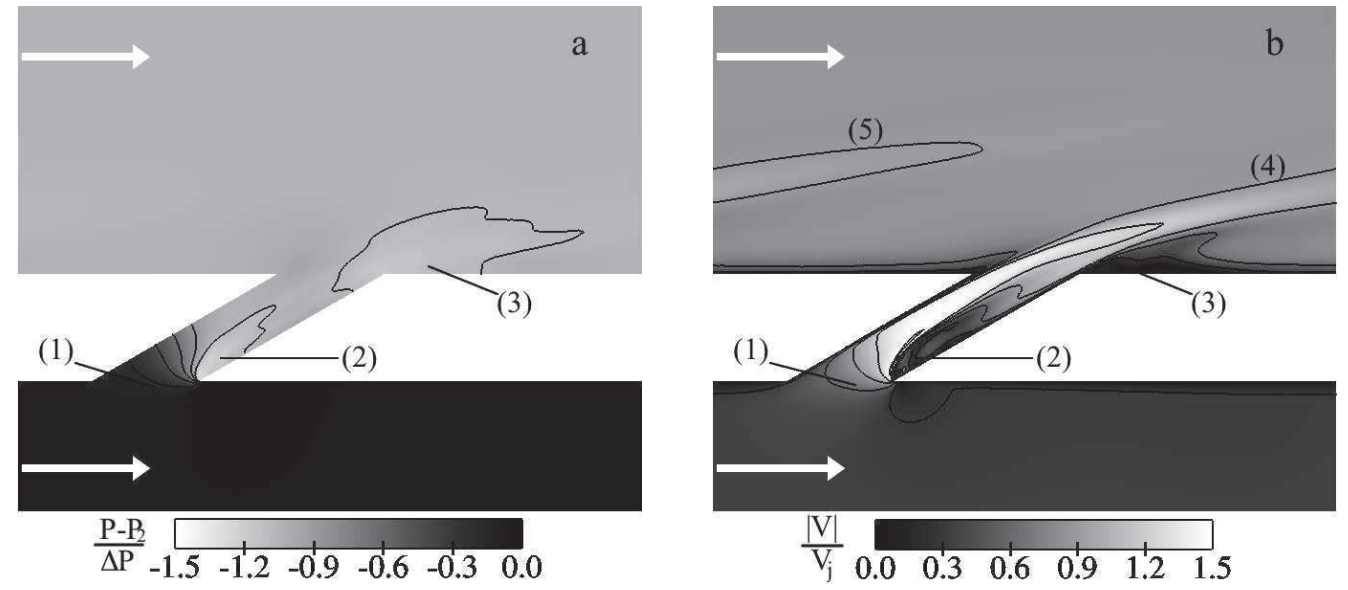

Figure 11. Time-averaged quantities from Run $\mathrm{C}$ on the centreline plane (zoom over the hole region) (a): Contours and isolines of the time-averaged pressure. (b): Contours and isolines of the time-averaged velocity magnitude $|V|$. White arrows show the flow direction in both channels.

hole: the jetting region along the upstream wall and the low-momentum region along the downstream wall of the hole. This structure is also reported by Brundage, Plesniak \& Ramadhyani (1999). When the jet issues in the upper channel, another separation zones is observed just downstream of the jet, near the wall (3). This separation is known to appear for relatively high blowing ratios and is responsible for a key feature of this type of flow, the entrainment phenomenon, visualised in $\S 4.4$ and 4.5.

Further in the primary main stream, the jet loses its strength by mixing with the main flow (4). Note that due to the periodic configuration, the jet that goes out of the domain reenters by the other side (5). Figure 11 strongly suggests that the shape of the micro-jet (1-4) is influenced by the aspiration side and that computing only the injection side would be questionable.

\subsection{Flow on the suction side}

Figure 12 presents the structure of the flow on the suction side by displaying, in a horizontal plane located $0.5 d$ under the suction wall, contours and isolines of the three components of the time-averaged velocity in figures $12(a-c)$ and contours of the $\mathrm{Q}$ criterion (Hunt, Wray \& Moin 1988) calculated from the time-averaged velocity in figure $12(d)$.

The acceleration of fluid entering the hole can be seen in figure $12(b)$, on the timeaveraged vertical velocity field, which is very inhomogeneous. The spatial-averaged vertical velocity over the horizontal plane is $0.02 V_{j}$ but locally in the cutting plane, it reaches $0.3 V_{j}$. Note also that the maximum of the vertical velocity is not centred under the hole inlet but is located downstream of the centre. This can be related to the pressure gradients observed in figure 11(a): the maximum pressure variations are observed at the downstream edge of the hole inlet.

The suction through the hole influences the three components of the velocity: figure 12(a) shows its effect on the streamwise velocity: under the upstream edge of the hole, the aspiration induces a small acceleration and under the downstream edge of the hole, a deceleration. Near the plate, negative values of the streamwise velocity are even observed, showing that the fluid turns back to enter the hole. The aspiration makes the fluid come from all sides of the hole, as observed on the time-averaged spanwise velocity field (figure 12c), which shows how the fluid comes from lateral sides. The streamwise 

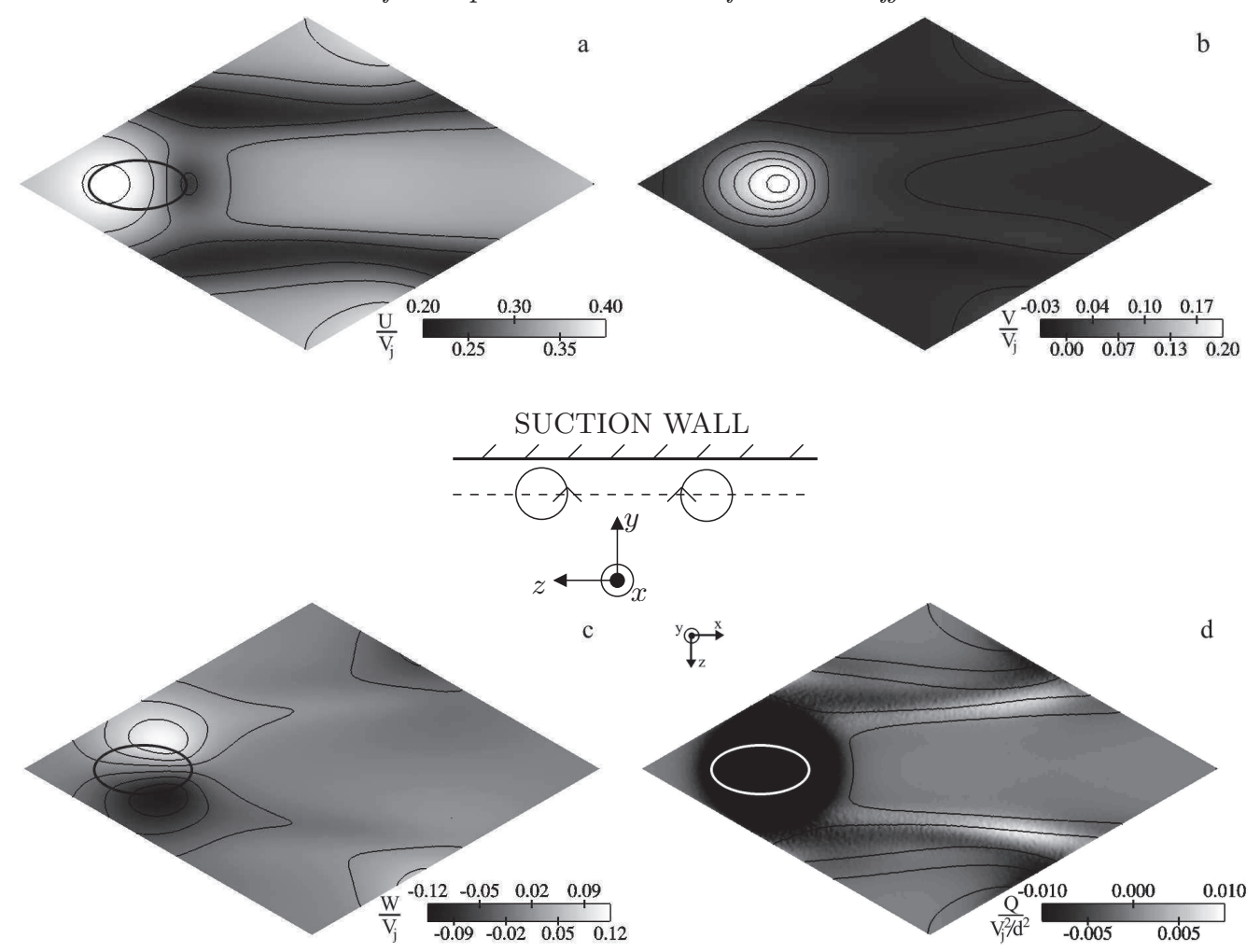

FiguRE 12. Time-averaged solution from Run $\mathrm{C}$ over a cutting plane located in the suction side at $0.5 d$ below the plate. The thick black/white ellipses correspond to the projection of the aperture inlet. (a): Contours and isolines of time-averaged streamwise velocity. (b): Contours and isolines of time-averaged normal velocity. (c): Contours and isolines of time-averaged spanwise velocity. (d): Contours of Q criterion. Isolines of time-averaged streamwise velocity as in (a). A schematic in the centre of the figure shows the direction of rotation of the vortices; the dotted line shows the location of the cutting plane $y=-2.5 d$ displayed in this figure.

velocity field also shows the presence of two bands of low velocity on each side of the hole. The lateral aspiration visualised in figure $12(c)$ creates a velocity deficit on both sides of the hole. Figure $12(d)$ presents isocontours of $\mathrm{Q}$ criterion (Hunt et al. 1988). This criterion, based on the second invariant of the velocity gradient tensor, is used to locate vortical structures: when the $\mathrm{Q}$ criterion is positive, the rotation rate is superior to the strain rate. In figure $12(d)$, positive values of the $\mathrm{Q}$ criterion are observed downstream of the hole. Two counter-rotating streamwise vortices are created at the lateral edges of the hole: their distance to the suction wall is approximately $0.5 d$. A schematic in the middle of figure 12 shows the direction of rotation of the vortices. Downstream of the perforation, the spanwise spacing between the vortices increases and they slightly move away from the suction wall. This vortical structure has already been reported both experimentally and numerically in MacManus \& Eaton (2000), where their formation process is detailed. Figures 12(a,d) also show that the streamwise vortices delimitate the low streamwise velocity zones (shown by isolines).

The flow near the perforated plate on the suction side proves to be highly threedimensional, with streamwise vortices appearing downstream of the perforation. This organisation is then very different from an idealised concept of a uniform suction. 


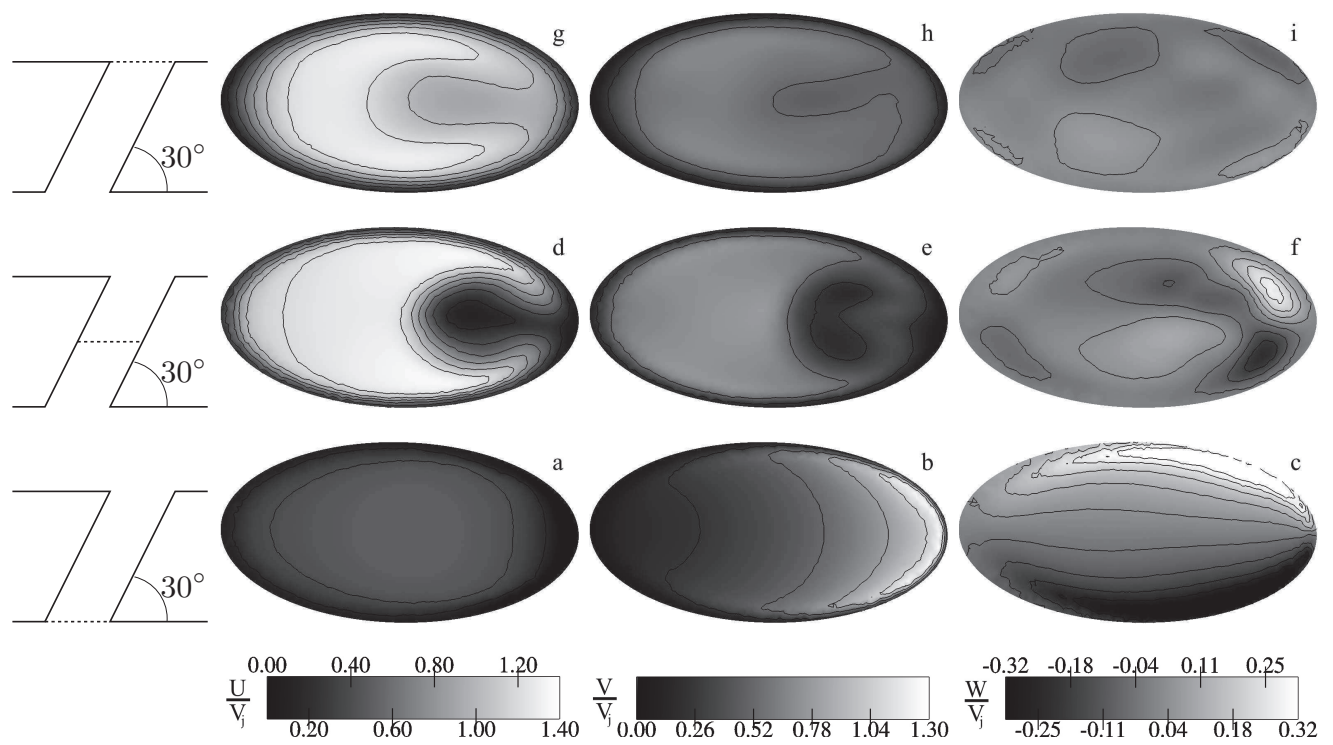

FIGURE 13. Contours and isolines of the three components of the time-averaged velocity on horizontal planes in the hole from Run C. The planes are represented on the left (angles are not conserved). Top row: outlet plane. Intermediate row: half-height plane. Bottom row: inlet plane. Left column: streamwise velocity. Central column: normal velocity. Right column: spanwise velocity.

\subsection{Flow within the aperture}

The flow inside the hole is known to be highly inhomogeneous. Information about the in-hole flow has been obtained through numerical simulations, either by RANS (Walters \& Leylek 2000) or LES (Iourokina \& Lele 2006). Due to the difficulty of performing direct measurements in the hole itself, experimental data are rare (see for example the work by the group of M. W. Plesniak for short normal holes, Peterson \& Plesniak 2002, 2004a).

Figure 13 shows contours and isolines of the three components of the time-averaged velocity over three horizontal planes from the inlet $(y=-2 d$, bottom row) to the outlet of the hole ( $y=0$, top row) from Run C. At the inlet of the hole (figure 13, bottom row), the flow is very similar to the one described in the former section devoted to the suction side of the plate. The streamwise velocity (figure 13a) is rather homogeneous in the inlet plane, with small values still related with the suction crossflow velocity. The vertical velocity field (figure $13 b$ ) is different. It shows small values at the upstream part of the hole inlet and high values (superior to $V_{j}$ ) near the downstream edge. As said before, this is related to the pressure field shown in figure 11(a): the strongest pressure gradients are observed near the downstream edge of the hole outlet, in the vertical direction. It induces a strong separation near the downstream wall of the hole. As seen on the organisation of the flow on the suction side, the aperture is fed by fluid particles coming from its lateral neighborhood: this explains the spanwise velocity field in figure 13(c), with strong values near the lateral edges of the hole.

In the middle of the hole (middle row), the flow is completely different. The upstream wall of the hole blocks the fluid that has, at the inlet of the hole, a strong vertical velocity and forces the jet to align in the direction of the hole. The jet is then flattened against the upstream wall, with smaller values of velocity magnitude near the downstream boundary. The vertical velocity is more homogeneous (figure 13e) than at the inlet (figure 13b). On the contrary, the streamwise velocity (figure $13 d$ ) shows a partic- 


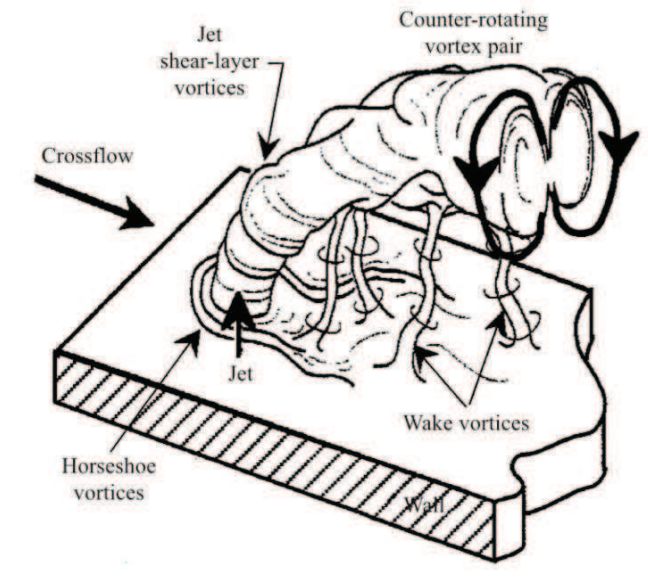

FigURE 14. Structural features of canonical wall-normal JCF (from Fric \& Roshko 1994).

ular form, characteristic of effusion cooling, the jet having a kidney shape. The jetting region and the low-momentum region defined by Walters \& Leylek (2000) (see also §4.1) are clearly observed in figure $13(d)$ and $13(e)$. The vertical and the spanwise components of the velocity allow observation of another characteristic of the velocity field in the hole: counter-rotating vortices appear in the aperture itself, in the low-momentum region. This type of organisation has often been reported before, for example by Leylek \& Zerkle (1994) or Brundage et al. (1999). The counter-rotating vortices seem to be related to the deformation of the velocity field due to the separation at the entry of the hole. Near the upstream wall, another pair of vortices can be seen. They are much less intense than the vortices observed in the low-momentum region and spanwise velocity values near the upstream wall are small. This structure is due to the FCFC configuration: it results from the aspiration of fluid experimenting a small spanwise movement on the suction side, due to former aspiration. It is thus due to the multiple hole geometry. This feature is expected to be stronger as the suction rate (ratio between the bulk vertical velocity and the crossflow velocity on the suction side) increases.

The structure of the flow does not change much between the half-height plane and the outlet of the hole (top row). Note however that the kidney shape is even clearer in the streamwise velocity (figure $13 \mathrm{~g}$ ) and the vertical velocity (figure $13 \mathrm{~h}$ ) is more homogeneous. The in-hole counter-rotating vortices do not appear as strong as within the hole. Indeed, they do not really survive when they reach the outlet of the hole.

The description of the flow in the hole shows that it is highly inhomogeneous. Such observations raise some questions about the validity of studies where the calculation domain is cut at the outlet or even at the inlet of the hole, imposing a particular velocity profile. In addition, in the context of cooling, the complexity of the flow compromises the use of simple correlations to assess the convective heat flux along the hole, an important data for the thermal design of combustion chambers.

\subsection{Vortical structure of the flow}

Before describing the velocity field near the wall on the injection side of the domain, the vortical topology of the flow is presented and compared with the classical jet-incrossflow structure. JCF configurations are dominated by coherent structures that have been abundantly studied in the literature. Many studies (Andreopoulos \& Rodi 1984; Cortelezzi \& Karagozian 2001; Fric \& Roshko 1994; Kelso, Lim \& Perry 1996; Muppidi 
\& Mahesh 2007) deal with the characterisation of the vortical structure of the flow in the case of large injection rate in the direction normal to the wall. As illustrated in figure 14, four different structures are usually reported in the instantaneous fields for canonical JCF: the counter-rotating vortex pair, the jet shear layer vortices, the horseshoe vortices and the wake vortices. The counter-rotating vortex pair is the main structure of the jet in crossflow: it is present in the far field, where it is aligned with the jet. The shear layer vortices result from the Kelvin-Helmholtz instability that develops at the edge of the jet. Horseshoe vortices are created by the adverse pressure gradient encountered by the primary main flow in the wall region, the jet acting as an obstacle for the crossflow. A wake region is observed downstream of the jet, with wall-normal orientated wake vortices starting from the wall and ending in the jet. The counter-rotating vortex pair and the horseshoe vortices are present in the average field. More recently, time-averaged wake vortices have been detected just downstream of the hole exit, both experimentally by Peterson \& Plesniak (2004a) and numerically by Hale, Plesniak \& Ramadhyani (2000) or Peet (2006). Peterson \& Plesniak (2004a) refer to these vortices as downstream spiral separation node vortices to distinguish them from the unsteady 'wake' vortices reported by Fric \& Roshko (1994).

Figure 15 displays the different vortical structures that are present in the simulations, in a time-averaged sense. The solid wall is partly transparent to allow observation of the hole and of the suction side. The main flow structure is the counter-rotating vortex pair (1), which dominates the wake of the jet. The two vortices originate from the lateral edges of the hole outlet, as observed experimentally by Gustafsson (2001) or numerically by Renze et al. (2006). Their direction of rotation is such that the fluid is pulled away from the wall at the centreline and entrained towards the wall when coming from the sides of the jet. As discussed in $\S 4.3$, two counter-rotating vortices aligned with the jet are also present in the hole itself (2), in the low-momentum region of the perforation. Even if the direction of rotation is the same for the aperture vortices and the main counter-rotating vortex pair (CVP), they do not form a unique structure. The second counter-rotating pair detected in the hole $(\S 4.3)$ is much less intense and is not observed in figure 15. A horseshoe vortex (3) is also observed just upstream of the hole. This classical structure for jets in crossflow is not reported in every similar works on inclined jets: Peet (2006) observes such a structure but it seems absent in Gustafsson (2001) and Tyagi \& Acharya (2003). Compared to canonical jets in crossflow, this structure is much weaker, consistently with the fact that the adverse pressure gradient experienced by the primary stream is smaller when the jet is inclined. The inclined jet does not block the incident flow as much as the normal one does. As stated by Bergeles et al. (1977), inclined jets induce substantial disturbances of the flow, but essentially downstream.

Two small vortices are detected just downstream of the hole exit: the downstream spiral separation node vortices (4). They originate from the wall, where they are almost vertical, and they are rapidly reoriented in the direction of the jet. Their other extremities rotate in the same direction as the counter-rotating vortex pair with which they coalesce. The vortices detected on the suction side (see figure 12) are also visible in this figure (5). The intensity of this vortex pair is small compared to the other structures displayed in figure 15. A secondary counter-rotating vortex pair (6) is observed under the primary CVP, with a direction of rotation opposite to that of the CVP. They are located very close to the wall and their direction is almost horizontal. This structure has been reported both numerically by Hale et al. (2000) or Yuan, Street \& Ferziger (1999) and experimentally by Andreopoulos \& Rodi (1984) or Kelso et al. (1996) for example, although their size and their proximity to the wall make their observation more difficult than the primary CVP. As for Kelso et al. (1996), wall vortices are steady and can be observed in instantaneous 


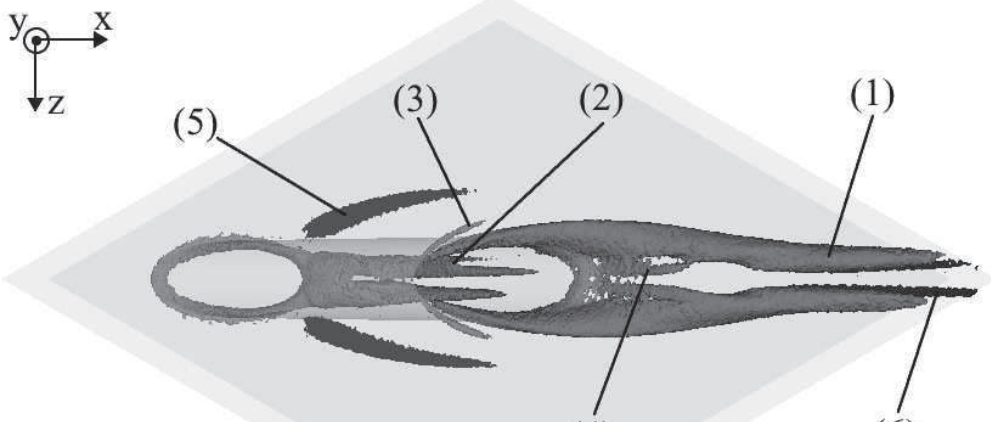

(4)

(6)
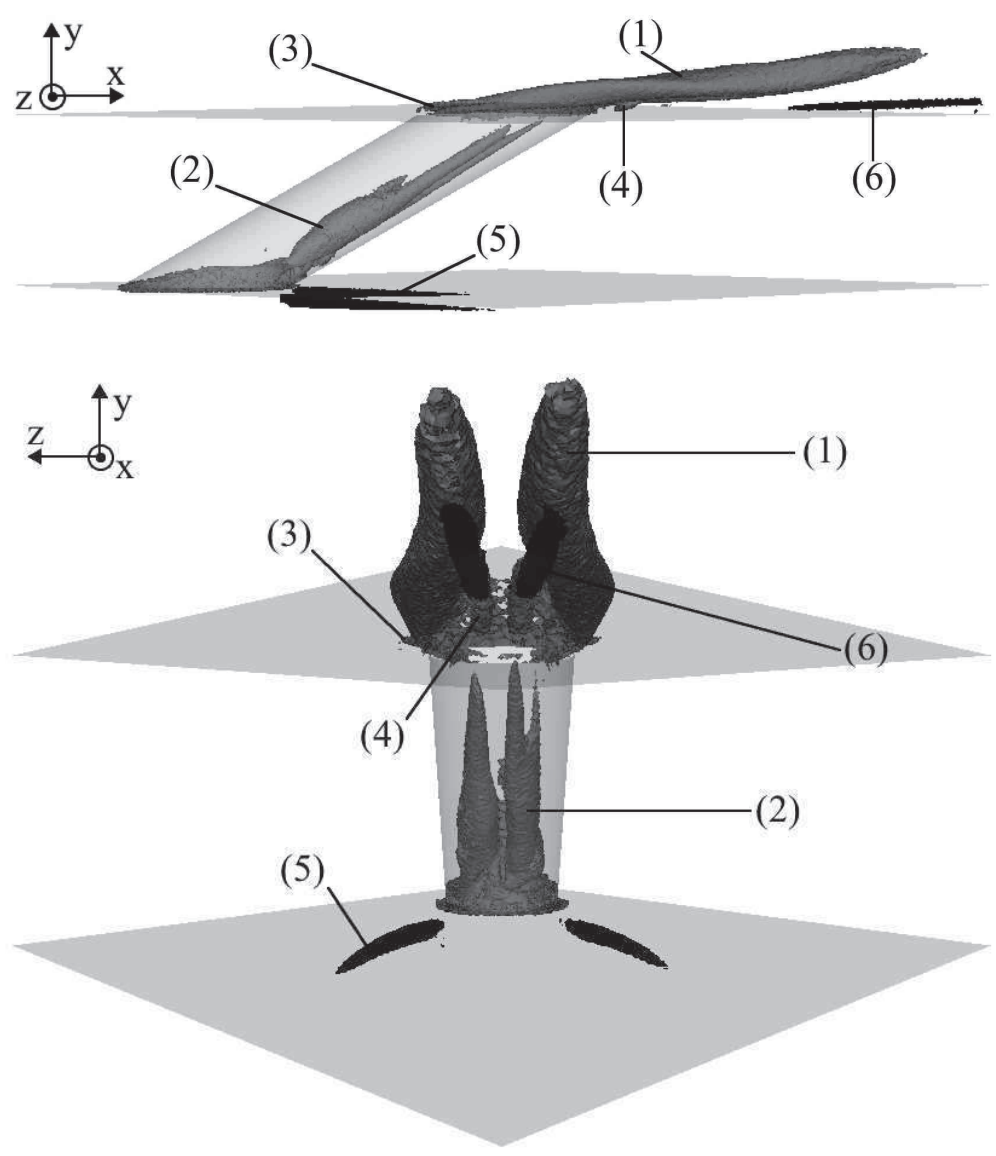

Figure 15. Grey isosurface of $\mathrm{Q}$ criterion $\left(0.55 V_{j}^{2} / d^{2}\right)$ from Run $\mathrm{C}$ showing four structures present in the time-averaged field: two pairs of counter-rotating vortices downstream of the hole (1) and within the aperture (2), the horseshoe vortex (3) just upstream of the hole, the small downstream spiral separation node vortices immediately downstream of the aperture exit (4). Two dark grey iso-surfaces of Q-criterion show two additional structures: the pair of suction vortices (5), $Q=0.05 V_{j}^{2} / d^{2}$ and small streamwise vortices (6) on the injection side, lying beneath the CVP, $Q=0.2 V_{j}^{2} / d^{2}$. 

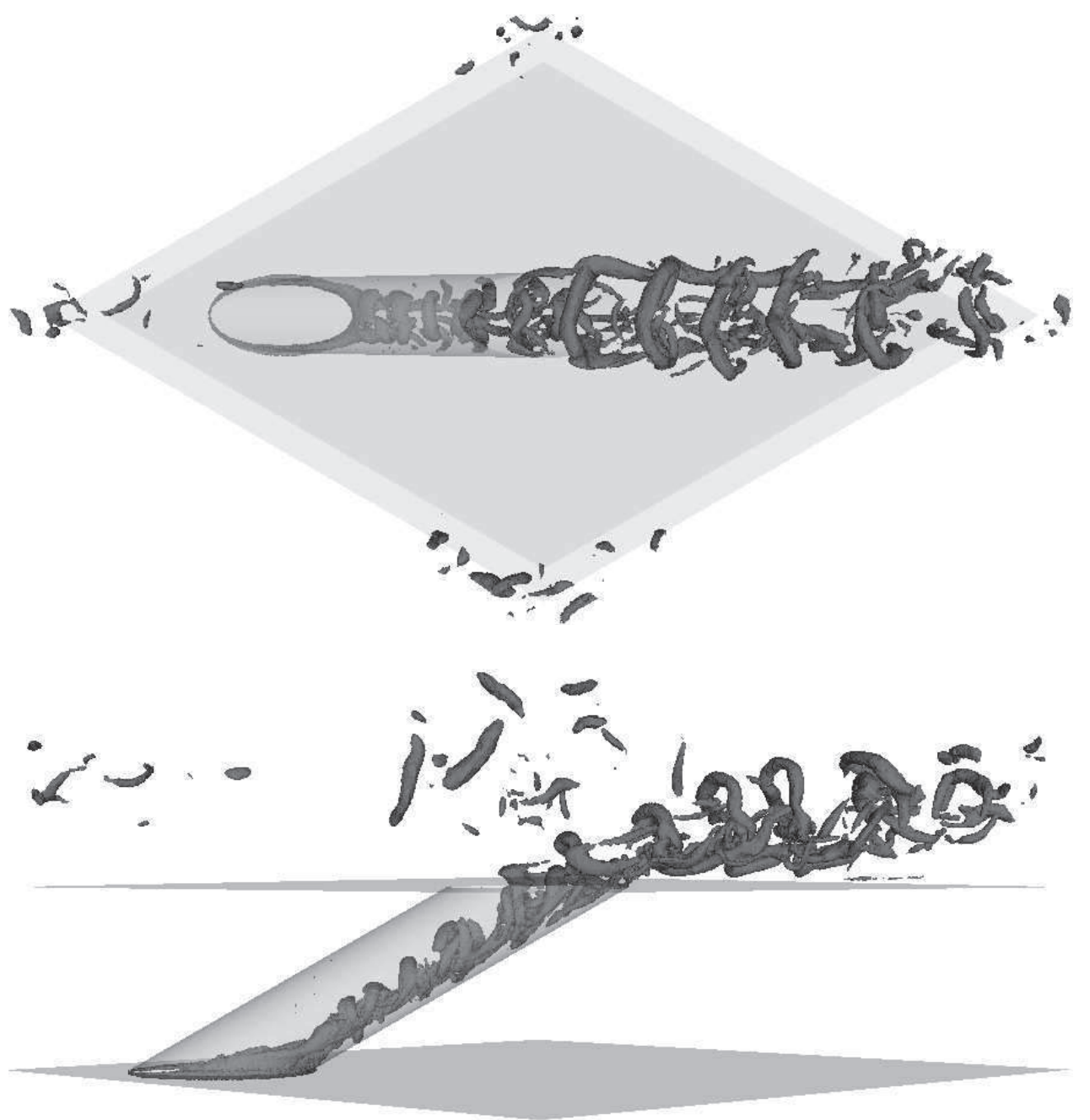

FiguRE 16. Isosurface of Q criterion $\left(5 V_{j}^{2} / d^{2}\right)$ from Run C showing the instantaneous flow structure. Top: top view. Bottom: side view.

fields. Note that they appear beneath the location where the vortices composing the CVP get closer from each other, at approximately $x=3.5 d$.

Instantaneous fields of Q-criterion offer a very different picture of the vortical flow structure. Instantaneous structures are much intense, as shown in figure 16, where an instantaneous iso-surface of Q-criterion at $5 V_{j}^{2} / d^{2}$ is shown. Again, the solid wall is partly transparent to allow observation of the in-hole behaviour. The instantaneous field is dominated by shear layer vortices formed at the upstream face of the jet and also inside the aperture, at the high shear zone that separates the low-momentum and the jetting regions. These in-hole vortices have an hairpin form with two legs located in the low-momentum region, in the direction of the hole. The counter-rotating vortex pair observed within the hole in the average field actually results from the averaging of series of hairpin vortices: they form a unique structure in an instantaneous visualisation. The hairpin vortices seem to result from the roll-up of the vorticity located at the sharp edge of the hole entrance. Outside the hole, in addition to the shear layer vortices, another type of vortices are detected. They are located close to the wall and have a streamwise 
orientation. Their direction of rotation corresponds to the one of the counter-rotating vortex pair observed in the time-averaged field (figure 15). In the instantaneous solution displayed in figure 16, they are more intense in one side of the jet $(z<0)$. They show the same spatial periodicity as the shear layer vortices (see figure 16, top view).

Figure 17 displays the spanwise vorticity field over the centreline plane, at eight different instants. Two images are separated by $\delta t=0.0156$ FTT. Two shear layers with negative spanwise vorticity (black colours in figure 17) are observed: the in-hole one separates the low-momentum and the jetting regions, and the leeward one is between the jet and the separation downstream the aperture. The windward shear layer exhibits positive values of spanwise vorticity. The in-hole shear layer destabilises and produces the in-hole hairpin vortices observed in figure 16. The Strouhal number of the in-hole structures, based on the vorticity thickness of the shear layer and the velocity difference is 0.14 . The vortices are convected by the effusion flow (see the black arrows in figure 17) and they destabilise the windward shear layer at the same frequency of $1640 \mathrm{~Hz}$. Small vortices with positive vorticity are then shed (white arrows); the Strouhal number in the windward shear layer is 0.54 . The triggering of the windward vortices by the in-hole structures that are initiated near the hole inlet demonstrates that computing both sides of the wall is necessary to capture the FCFC flow physics. The in-hole vortices are convected outside the aperture but they are rapidly dissipated: none can be observed downstream of $x \approx 1.2 d$. Note also that the leeward shear layer does not form any coherent structure. As a consequence, only structures with positive spanwise vorticity are observed outside the hole, on the injection side. This is different from what observed Tyagi \& Acharya (2003) in their LES, in a comparable configuration, with a blowing ratio equal to unity. These authors interpret the vorticity fields from their computation as traces of hairpin structures shed from a thick shear layer observed in the downstream wall of the hole. Instead, the present results show that the shear layer vortices and the CVP form two different structures (figure 16). The slightly lower blowing ratio, the insufficient grid resolution and smaller computational domain (starting at the hole inlet) used in Tyagi \& Acharya (2003) may explain these differences. Figure 17 also shows that the separation zones are regions of intense activity.

\subsection{Flow organisation on the injection side: jet and wake}

In order to show how the jet behaves after having penetrated the crossflow, figure 18 displays contours and isolines of the time-averaged streamwise velocity $\langle U\rangle$, the streamwise RMS velocity $u_{\mathrm{rms}}$ and the vertical RMS velocity $v_{\mathrm{rms}}$ in the mid plane $z=0$ and the time-averaged vertical velocity $\langle V\rangle$ in a plane normal to the crossflow direction and located three diameters downstream of the hole centre. The black line in figure 18( $a)$ represents the trace of the cutting plane displayed in figure $18(d)$.

Just after the outlet (1), the jet bends due to the crossflow (figure 18a) and separates from the wall. As reported in $\S 4.1$, a zone of low velocity can be observed (2). Just downstream of the separation zone, under the jet, the velocity increases (3) because of the bypass of the jet by the main flow: part of the main flow is entrained between the jet and the wall. Figure 18(b) displays the variations of the streamwise RMS velocity. In the zone of strong shear in the hole (4), high levels of fluctuations are observed; they are mainly due to vortex shedding displayed in figure 17. In the wake of the jet, a second zone of high fluctuations can be noticed (5). This is also a region of strong shear, between the jet core and the separation zone. In this region, velocity fluctuations are mostly due to the variation of the jet position. Note that on the upstream side of the jet, the fluctuations are much smaller. Inclined jets in crossflow with moderate blowing ratio often show this type of behaviour because the velocity of the jet is close to that of the crossflow (Peet 

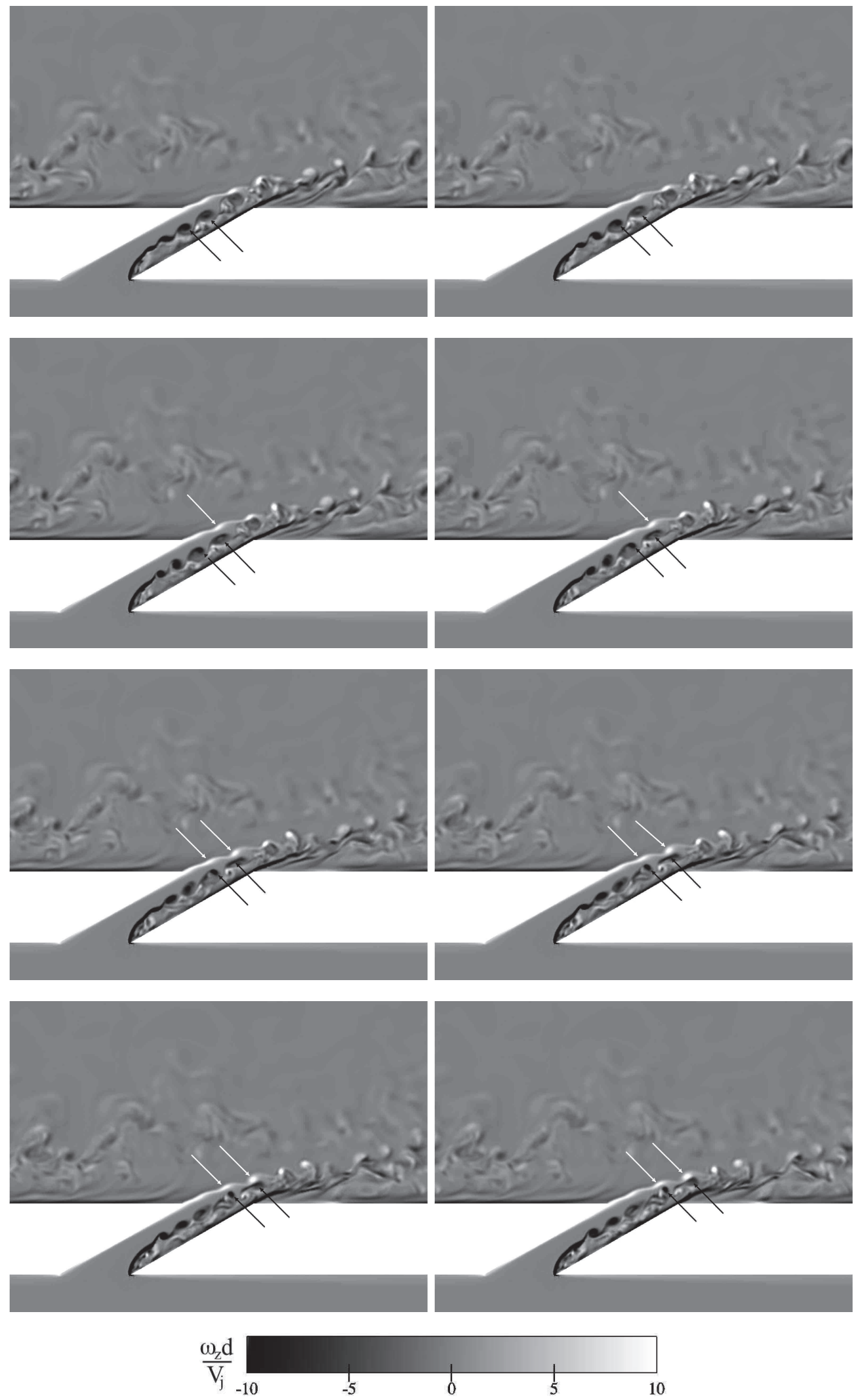

FIGURE 17. Instantaneous views of the spanwise vorticity field $\omega_{z}$ over the centreline plane. Reading is from left to right and top to bottom. Two images are separated by $\delta t=0.0156$ FTT. The black arrows follow two consecutive in-hole vortices with negative vorticity; the white arrows show the two corresponding positive vortices triggered in the windward shear layer. 

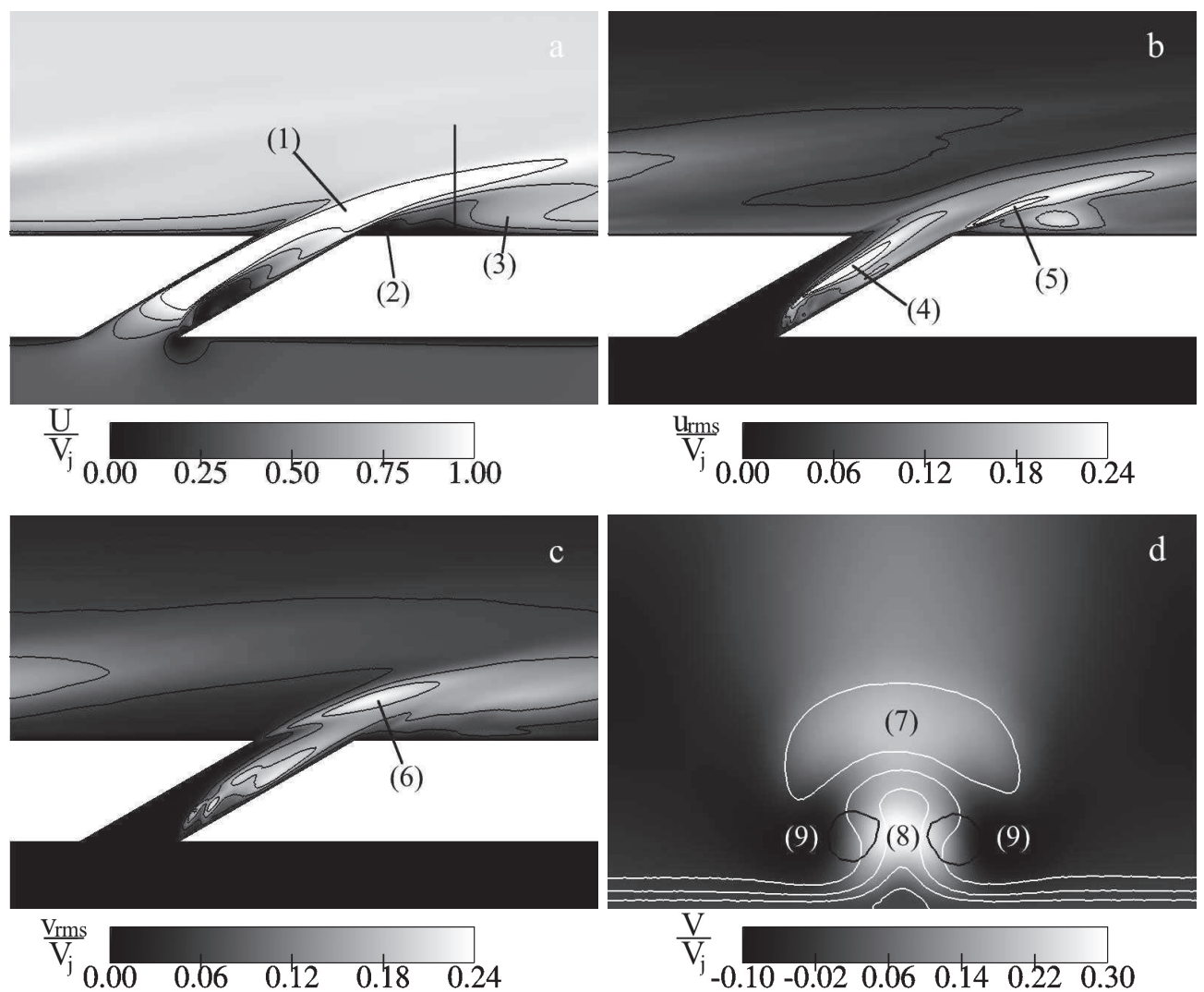

Figure 18. Description of the jet and its wake. (a): Contours and isolines of the time-averaged streamwise velocity on the mid plane $z=0$. (b): Contours and isolines of the streamwise RMS velocity on the mid plane $z=0$. (c): Contours and isolines of the vertical RMS velocity on the mid plane $z=0$. (d): Contours of the time-averaged vertical velocity on a plane normal to the direction of the crossflow, visualised in (a) by a black vertical line ( $x=3 d$, zoom on the near-wall region). White isolines of the time-averaged streamwise velocity (same values as in a). Black isolines of the $\mathrm{Q}$ criterion $\left(Q=V_{j}^{2} / d^{2}\right)$ to show the counter-rotating vortex pair.

2006). Figure 18(c) displays the field of vertical RMS velocity in the mid plane $z=0$. High levels of velocity fluctuations are observed in the in-hole separation zone and at the windward edge of the jet (6).

Figure $18(d)$ shows the contours of the time-averaged vertical velocity. Black isolines of Q criterion $\left(Q=V_{j}{ }^{2} / d^{2}\right)$ are used to locate the two main vortices of the wake. White isolines of time-averaged streamwise velocity allow observation of the location of the jet core. From figure 18(d), the jet (7) conserves the kidney shape it has in the hole (see $\S 4.3)$ after it penetrates the main stream. However, it is no longer confined by the walls of the hole and it becomes wider (approximately $1.6 \mathrm{~d}$ ). The two counter-rotating vortices forming the CVP (see figure 15) are located under the jet, and the distance between their centres is approximately $0.6 \mathrm{~d}$. They induce a zone of low streamwise and high vertical velocity between them (8). Figure 18( $d)$ also shows how the main flow is convected near the wall (negative vertical velocity is observed on both sides of the jet (9)) and then decelerated in the streamwise direction (8) under the effect of the two counter-rotating vortices under the jet. Note that the vertical velocity under the jet (8) is actually larger than in the jet itself (7). These features are related to the entrainment effect, responsible for the whole structure of the film. In non-isothermal cases, at the beginning of a cooling 
film, the main flow is only composed by hot gases that are entrained towards the wall. This explains the classical results of very bad overall efficiency observed for high blowing ratios at the beginning of the film or for isolated jets (see for example Rouvreau 2001). On the contrary, far from the leading edge of the multi-perforated plate, the entrainment phenomenon reattaches the cool air coming from previous jets, forming a robust film that really isolates the plate, even downstream of the zone where the plate is perforated (Mayle \& Camarata 1975; Yavuzkurt et al. 1980b).

The centreline jet trajectory can be evaluated by tracking the location of maximum jet velocity. The jet trajectory is displayed in figure 19, together with the correlation proposed by Ivanov (1963) for canonical inclined JCF. This correlation has been tested at various angles and blowing ratios by Margason (1968) and seems a robust correlation for trajectories of inclined JCF:

$$
\frac{x}{d}=\left(\frac{1}{R}\right)^{2.6}\left(\frac{y}{d}\right)^{3}+\frac{y}{d} \cot \alpha_{g}
$$

where $\alpha_{g}$ is the geometrical angle of the perforation and the most natural choice for the jet-to-mainstream velocity ratio is $R=M_{b}=1.17$ (see table 4 ). This correlation is compared to the trajectory obtained from Run C in figure 19. Note that the trajectories do not meet the origin because the location of the maximum of velocity magnitude at the hole exit is located $x=0.42 d$ upstream of the hole centre. The numerical jet trajectory has the usual shape for JCF. However, its curvature is smaller than the classical one for JCF and the long distance behaviour is $x / d \propto(y / d)^{2.5}$ instead of $(y / d)^{3}$ in the JCF correlation. The two trajectories cross at $x / d \approx 7$ and the most significant differences appear for long distances from the hole: the LES trajectory is approximately half a diameter higher than the correlation for $x / d \approx 15$. Several reasons might explain these differences in the trajectories:

(a) the effective flow angle slightly departs from the geometrical angle (see $\S 5.2$ ). Note however that the $\cot \alpha_{g}$ term is not the main term for long streamwise distances in equation 4.1 ,

(b) because of the FCFC configuration, the mainstream is affected by the previous upstream jets before it interacts with the current one. Recall that due to the effect of the main CVP, the streamwise velocity is smaller downstream the (upstream) jet (see figure 18d). Thus the velocity of the effective mainstream seen by the current jet is smaller than in the case of a single JCF with the same outer conditions. As a direct consequence, the penetration is somewhat more effective. This effect has been assessed in figure 19 where the correlation 4.1 has been also plotted for $R=1.25$, which corresponds to the bulk streamwise velocity computed at $x=-2 d$ over the ranges $0<y<2 d$ and $-d<z<d$,

(c) because of the small plate thickness-to-diameter ratio, the effusion flow within the aperture is not established (see $\S 4.3$ ). The flow behaviour in the aperture is known to have an effect on jet trajectory, as shown by Peterson \& Plesniak (2002): the in-hole vortices rotating in the same direction as the CVP reinforces this structure, inducing a higher trajectory,

(d) because of the bypass of the previous upstream jets, the vertical velocity of the main flow interacting with the current jet is not negligible. In the present case, it is of order $6 \%$ of the streamwise velocity. This characteristic of the mainstream has most probably the effect of limiting the bending of the jet, consistently with a curvature smaller than for the JCF case,

(e) as depicted in figure 19, the jet-to-jet downstream distance might be small enough so that the downstream jet generates an additional lift-off of the trajectory by inflecting 


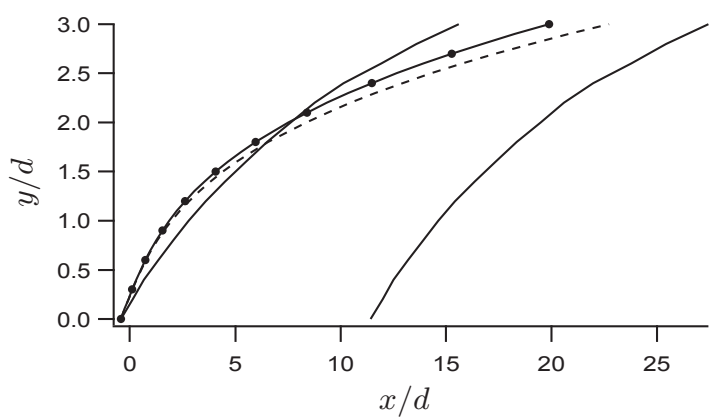

Figure 19. Jet trajectory from Run $\mathrm{C}(-)$. Comparison with the correlation of Ivanov (1963) with $R=M_{b}(---)$ ) and $R=1.25$ ( - - - ).

the main stream, at least for $x / d \geq 12$ (recall that the hole-to-hole streamwise distance is $11.68 \mathrm{~d})$,

$(f)$ due the staggered arrangement of the holes, the penetration of the current jet might be enhanced by the presence of the two lateral jets located downstream, at half the downstream hole-to-hole distance.

Note that the first two items can be accounted for in equation 4.1 by tuning the velocity ratio and flow angle. Figure 19 shows that the penetration of the jet is better reproduced by the JCF correlation when $R=1.25$ is used instead of $R=M_{b}$. The last four items are related to the presence of regions with non negligible vertical velocity beside the current jet. Specific to FCFC cases, they are also consistent with the smaller curvature and deeper penetration of the jet observed in figure 19.

\section{Discussion}

In this section, the LES results are analysed to provide information about the wall fluxes modelling on both sides of the perforated plate. In $\S 5.1$, the fluxes at the wall are post-processed from Run $\mathrm{C}$ in order to determine the most important contributions. In $\S 5.2$, for each side of the plate, an attempt to model the main contribution of the streamwise momentum flux is presented.

\subsection{Wall fluxes}

The perforated plate is a combination of holes and solid wall. At the suction side, the liner can be seen as a solid wall plus an outlet and at the injection side, as a solid wall plus an inlet. The fluxes are then a combination of inlet/outlet fluxes and solid wall fluxes. The configuration tested in this paper being isothermal, only the momentum fluxes are considered in the remainder of this section.

The wall fluxes for the three components of the momentum have been post-processed from Run $\mathrm{C}$ and are presented in tables 5, 6 and 7. Each flux at the wall is decomposed into contributions from the hole outlet/inlet (surface $S_{h}$ ) and from the solid wall (surface $S_{s}$ ) and also into viscous and non-viscous parts. The relative importance of each contribution in the total flux at the wall can be assessed from these tables. Note that viscous contributions are not presented in table 6 , as they are negligible compared to non-viscous terms. Subscripts 1, 2 and 3 correspond to the three coordinates $x, y$ and $z$. The outgoing normal to the wall is $\boldsymbol{n}$. In the case of interest, $\boldsymbol{n}$ has only a vertical component: $n_{2}=-1$ for the injection wall and $n_{2}=1$ for the suction wall. $\tau_{i j}$ is the 


\begin{tabular}{|c|c|c|c|c|}
\hline Region & total plate & hole & & solid wall \\
\hline Expression & $\int_{S}\left(<-\rho U V+\tau_{12}>\right) n_{2} d s$ & $\int_{S_{h}}-<\rho U V>n_{2} d s$ & $\int_{S_{h}}<\tau_{12}>n_{2} d s$ & $\int_{S_{s}}<\tau_{12}>n_{2} d s$ \\
\hline $\begin{array}{l}\text { Injection } \\
\text { Suction }\end{array}$ & $\begin{array}{r}7.21 \times 10^{-1} \\
-2.83 \times 10^{-1}\end{array}$ & $\begin{array}{c}114.1 \\
86.8\end{array}$ & $\begin{array}{c}-0.1 \\
0.0\end{array}$ & $\begin{array}{c}-14.0 \\
13.2\end{array}$ \\
\hline
\end{tabular}

TABLE 5. Time averaged wall fluxes for streamwise momentum from Run C: First column: expression and values of the total flux (in $\rho_{j} V_{j}^{2} d^{2}$ ) on both sides of the plate (total surface $S$ ). Columns 2-4: relative contributions (in \%) of the terms involved in the wall fluxes.

\begin{tabular}{cccc}
\hline Region & total plate & hole & solid wall \\
Expression & $\left.\int_{S}<-P-\rho V^{2}+\tau_{22}>\right) n_{2} d s$ & $\int_{S_{h}}-<P+\rho V^{2}>n_{2} d s$ & $\int_{S_{s}}-<P>n_{2} d s$ \\
& & & 96 \\
Injection & $3.42 \times 10^{3}$ & 4 & 96
\end{tabular}

TABLE 6. Time averaged wall fluxes for vertical momentum from Run C: First column: expression and values of the total flux (in $\rho_{j} V_{j}^{2} d^{2}$ ) on both sides of the plate (total surface $S$ ). Columns 2-3: relative contributions (in \%) of the non-viscous terms involved in the wall fluxes.

\begin{tabular}{ccccc}
\hline Region & total plate & hole & & solid wall \\
Expression & $\int_{S}\left(<-\rho V W+\tau_{32}>\right) n_{2} d s$ & $\int_{S_{h}}-<\rho V W>n_{2} d s$ & $\int_{S_{h}}<\tau_{32}>n_{2} d s$ & $\int_{S_{s}}<\tau_{32}>n_{2} d s$ \\
& & 38.1 & -3.6 & 65.5 \\
Injection & $-1.21 \times 10^{-4}$ & 116.9 & -4 & -12.9
\end{tabular}

TABLE 7. Time averaged wall fluxes for spanwise momentum from Run C: First column: expression and values of the total flux (in $\rho_{j} V_{j}^{2} d^{2}$ ) on both sides of the plate (total surface $S$ ). Columns 2-4: relative contributions (in \%) of the terms involved in the wall fluxes.

viscous stress tensor:

$$
\tau_{i j}=\mu\left(\frac{\partial V_{i}}{\partial x_{j}}+\frac{\partial V_{j}}{\partial x_{i}}\right)-\frac{2}{3} \mu \frac{\partial V_{k}}{\partial x_{k}} \delta_{i j}
$$

where $\mu$ is the dynamic viscosity $\left(\mu=1.788 \times 10^{-5} \mathrm{~kg} \mathrm{~m}^{-1} \mathrm{~s}^{-1}\right), V_{i}(i=1,2,3)$ the velocity components, $x_{i}(i=1,2,3)$ the coordinates and $\delta_{i j}$ the Kronecker symbol. Recall that $<>$ denotes time averaged quantities.

Several statements can be made from tables 5 to 7 :

(a) streamwise momentum $\langle\rho U>$ : the non-viscous streamwise momentum flux (table 5 , second column) is the main term for both the suction and the injection sides of the perforated plate. The viscous term over the hole surface is very small. The wall friction over the solid wall is approximately 8-10 times smaller than the non-viscous aperture term for the operating point considered. This means that one can only focus on the inviscid part of the flux when developing a first order model for effusion. In other words, assuming that the turbulent transfers scale as the wall friction, turbulence is not a firstorder issue when dealing with discrete effusion, which is of course significantly different from the classical case of an attached boundary layer over a solid plate,

(b) vertical momentum $\langle\rho V\rangle$ : the flux of normal momentum involves a pressure term that is clearly dominant. As pressure is almost constant, the repartition of fluxes between hole surface and solid wall surface corresponds to the porosity of the plate 


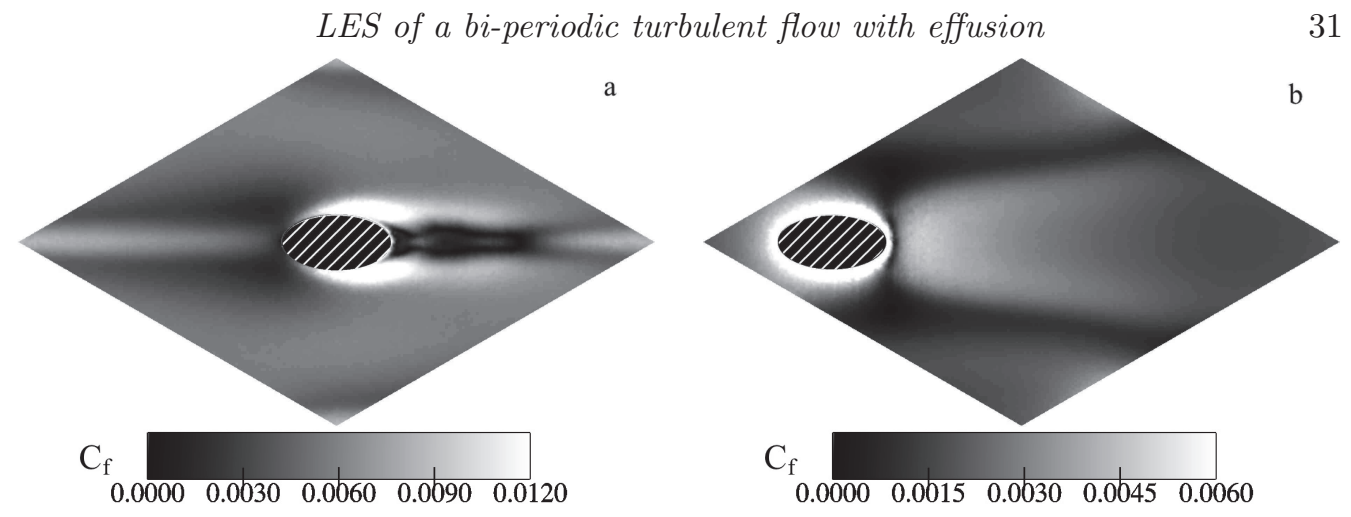

Figure 20. Time-averaged skin friction coefficient $C_{f}$ on both sides of the liner. The hole outlet/inlet is represented with a hatched ellipse. (a): View of the liner from the injection side. (b): View of the liner from the suction side.

$(\sigma \approx 4 \%)$. Note that the velocity term in the hole does not modify this repartition: it is small compared to the pressure term. This is true as long as the pressure drop is small compared to the operating pressure, which is the case in gas turbine applications,

(c) due to the symmetry of the problem, the spanwise momentum flux should be zero. The computation is almost symmetrical, the spanwise momentum flux being 1000 times smaller than the streamwise momentum flux.

Even if the porosity of the plate is small $(\sigma \approx 4 \%)$, table 5 shows that wall friction is not the main effect at the wall. However, the skin friction at the wall can be locally high, as shown in figure 20, where the distributions of the skin friction coefficient over the suction and injection walls are presented. The skin friction coefficient is $C_{f}=2 \tau_{w} /\left(\rho_{j} V_{j}^{2}\right)$, where $\tau_{w}$ is the total wall shear stress. As expected from the velocity field analysis, the friction at the wall is strongly inhomogeneous. On the injection side (figure 20a), the field of wall friction shows a structure corresponding to the characteristics of the flow described in $\S 4.5$ : upstream of the jet, the flow is lifted off and low values of skin friction coefficient are obtained. The primary counter-rotating vortex pair is observed thanks to the high values of wall shear stress. The CVP accelerates the flow near the wall, resulting in higher wall shear stress downstream of the aperture edges. Downstream of the jet separation, the velocity near the wall is small and low values of wall shear stress are observed. Just downstream of the hole, two lobes exhibit very low value of wall shear stress. This feature has to be related with downstream spiral separation node vortices (Peterson \& Plesniak 2004a,b). The structure of the wall shear stress is very similar to the one observed experimentally by Peterson \& Plesniak (2004b). Note however that due to the reattachment induced by the entrainment of the main flow towards the wall, the region of skin friction deficit stops 3.5 diameters downstream of the hole while it extends more than 10 diameters in the normal hole case (Peterson \& Plesniak 2004b). Downstream of the deficit region, traces of the secondary pair labelled (6) in figure 15 are detected in figure figure 20a. On the suction side (figure 20b), the presence of lowvelocity zones is also observed, with small values of wall shear stress. Maximum values are observed all around the hole inlet, where the acceleration of fluid induced by the aspiration is the strongest. Just downstream of the hole inlet centre, the fluid reattaches, showing high values of wall shear stress. These features are consistent with the flow structure described in $\S 4.2$. 


\begin{tabular}{|c|c|c|c|c|}
\hline Expression & $-\rho{\overline{\overline{U V}^{t}}}^{s} n_{2}$ & $-\rho \overline{\bar{U}}^{t} \overline{\bar{V}}^{t} n_{2}$ & $-\rho{\overline{\left(\bar{U}^{t}\right)^{s}\left(\bar{V}^{t}\right)^{s}}}^{s} n_{2}$ & $-\rho{\overline{\overline{(U)^{t}(V)^{t}}}}^{s} n_{2}$ \\
\hline Injection & $5.24 \times 10^{-1}$ & 89.2 & 10.8 & $<1 \%$ \\
\hline Suction & $-1.56 \times 10^{-1}$ & 109.6 & -9.6 & $<1 \%$ \\
\hline
\end{tabular}

TABLE 8. Decomposition of the non-viscous contribution of the streamwise momentum flux per unit surface from Run C: First column: values of the total contribution (in $\rho_{j} V_{j}^{2}$ ) on both sides of the plate. Columns 2-4: relative contributions (in \%) of the terms detailed in equation 5.4.

\subsection{Assessment of the non-viscous fluxes at the perforated plate}

A key step for the modelling of effusion cooling is to estimate mass/momentum/energy fluxes at both sides of the perforated wall. The amount of air passing through the holes is related to the pressure drop across the plate, through a discharge coefficient. The object of this section is not to propose an assessment of the discharge coefficient but to determine the momentum fluxes at the wall at a given mass flux through the hole.

In views of the results presented in tables 5 to 7 , assessing the vertical and the spanwise momentum fluxes is straightforward: the vertical momentum flux at the wall is directly related to the value of the pressure at the plate, a quantity that can easily be estimated in the RANS context from the pressure value at the first off-wall mesh point. As the perforation does not have any spanwise orientation, the spanwise momentum flux at both sides of the plate is null. On the contrary, the streamwise momentum flux cannot be determined easily. In the remainder of this section, focus is made on the possibility to propose a rough estimation of the main contribution to this flux, viz. its inviscid part through the hole inlet/outlet. Note first of all that for any quantity $\phi(\mathbf{x}, \mathbf{t})$ that depends on both space and time, the following decompositions can be considered:

$$
\begin{aligned}
& \phi(\mathbf{x}, \mathbf{t})=\bar{\phi}^{t}(\mathbf{x})+(\phi)^{\mathbf{t}}(\mathbf{x}, \mathbf{t}) \\
& \phi(\mathbf{x}, \mathbf{t})=\bar{\phi}^{s}(t)+(\phi)^{s}(\mathbf{x}, \mathbf{t})
\end{aligned}
$$

where $-{ }^{t}$ is the time-averaging operator and $-^{s}$ denotes the spatial-averaging operator over the hole inlet or outlet surfaces $\left(S_{h}\right)$. Consistently, ()$^{t}$ and ()$^{s}$ denote the fluctuations from the time and spatial averages. Note eventually that $-{ }^{t}$ is nothing but the $<>$ operator used in the previous sections. Combining these two decompositions, the inviscid flux per unit hole surface can be written as (the mass density $\rho$ is supposed to be constant in space and time over the hole inlet/outlet surface):

$$
-\rho{\overline{\overline{U V}^{t}}}^{s} n_{2}=-\rho\left({\overline{\bar{U}^{t}}}^{s}{\overline{V^{t}}}^{s}+{\overline{\left(\bar{U}^{t}\right)^{s}\left(\bar{V}^{t}\right)^{s}}}^{s}+{\overline{\overline{(U)}^{t}(V)^{t}}}^{s}\right) n_{2}
$$

The first term is the product of time and spatial averaged quantities, the second one estimates the non-uniformity and correlation of time averaged velocity components over the hole surface. The third term is the spatial average of the classical Reynolds stress based on time averaging. The values of each of these terms are reported in table 8 , for the hole outlet (injection) and inlet (suction). A reasonable estimation of the non-viscous streamwise momentum flux per unit surface of aperture is obtained from the product of time and spatial-averaged quantities. Assessing $\overline{U V}^{s}$ by $\overline{\bar{U}}^{t} \bar{V}^{t}$ leads to an error of approximately $10 \%$. This difference is mainly due to the non-uniformity of the timeaveraged velocity field over the hole inlet and outlet surfaces. The term of fluctuations in time does not contribute in the mean although the turbulence activity is substantial, 


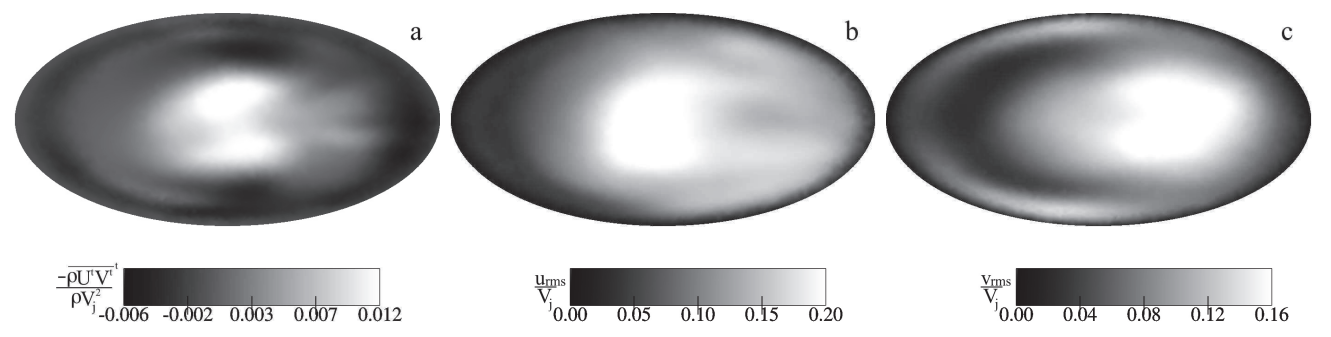

Figure 21. Reynolds stress and velocity fluctuations at the hole outlet. (a): Contours of the the Reynolds stress $-\rho{\overline{(U)^{t}(V)^{t}}}^{t}$, (b): Contours of the streamwise root mean square velocity, (c): Contours of the vertical root mean square velocity.

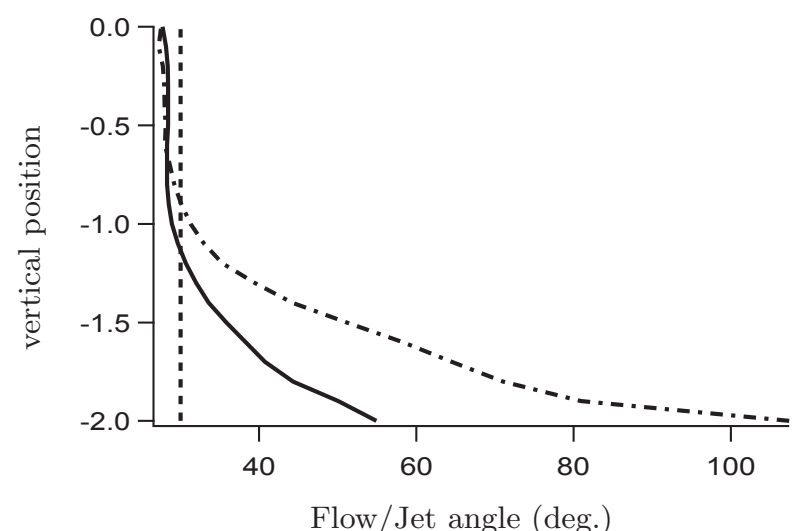

Figure 22. Flow angle ( - ) and jet angle ( - - ) as a function of the vertical position: $y=-2 d$ is the position of the hole inlet and $y=0$, the position of the hole outlet. The geometric hole angle $\left(\alpha_{g}=30^{\circ}\right)$ is also plotted $(---)$.

especially near the centre of the hole outlet where the turbulence intensity is as large as $30 \%$ : figure $21(b, c)$ shows that streamwise and vertical root mean square velocity can reach $20 \%$ of the bulk velocity in the hole. However, as shown in figure 21(a), the Reynolds stress $-\rho \overline{(U)^{t}(V)^{t}}$ is positive in the hole centre and negative in the wall region so that its contribution to equation 5.4 is very small.

In the previous approximation of the momentum flux, the ${\overline{\bar{V}^{t}}}^{s}$ term is easy to estimate, as the mass flow rate is supposed to be known. On the contrary, the time-space average over the hole inlet/outlet of the streamwise velocity is not known a priori. One way to proceed is to relate these two quantities via the flow angle $\alpha$ defined as the angle between the $(x, z)$-plane and the time-averaged, plane-averaged velocity vector. In other words, the flow angle is such that $\overline{\bar{V}}^{s}={\overline{\bar{U}^{t}}}^{s} \tan \alpha$ : if the plane-averaged velocity vector is vertical, the flow angle is $90^{\circ}$ and if it is along the streamwise direction, $\alpha=0^{\circ}$. Of course, a natural modelling idea would be to assume that $\alpha$ is imposed by the geometrical characteristics of the aperture (recall that the geometrical angle is $\alpha_{g}=30^{\circ}$ ). In order to test this simple idea, figure 22 shows the evolution of the flow angle in the hole as a function of the vertical coordinate (solid line). The hole inlet (suction wall) is located at $y=-2 d$ and the hole outlet (injection wall) is at $y=0$. The averaged orientation of the flow within the hole changes along the aperture and proves to be different from the hole angle. At the inlet of the hole, $\alpha$ is approximately $55^{\circ}$, almost twice as large as the 
geometrical angle. Over the first half of the row, the orientation of the flow progressively changes and is nearly aligned with the hole direction between $y=-1.2 d$ and $y=-0.4 d$ $\left(29^{\circ}\right)$. Near the hole outlet, the angle continues to decrease to reach $28^{\circ}$ at the hole outlet. Note that $\alpha$ is different from the classical definition of the jet angle, which is assessed at the location of maximum velocity magnitude, as the jet trajectory (see $\S 4.5$ ). The jet angle in the aperture is also reported in figure 22. The jet angle is close to $\alpha$ at the hole outlet $\left(27.5^{\circ}\right)$ but it is more than $90^{\circ}$ at the hole inlet. This is consistent with the pressure gradient at the hole inlet, which is almost vertical near the sharp edge of the hole, where the velocity magnitude is maximum.

It follows from figure 22 that the geometrical angle is not relevant to the flow behaviour near the hole inlet. On the contrary, the flow is almost aligned with the hole at the outlet: the streamwise velocity at the hole outlet is strongly related to the vertical velocity, through the hole angle, the angle mismatch being only $2^{\circ}$. Note however that this difference is not without consequence: the averaged streamwise velocity at the outlet plane $\overline{\bar{U}}^{s}$ would most likely be of interest in any model aiming at reproducing the momentum transfer through the plate. Assessing this quantity from the averaged vertical velocity at the outlet plane $\overline{\bar{V}}^{s}$ (easily assessed from the global injection mass flow rate) and the hole angle as $\overline{\bar{U}}^{s}=\overline{\bar{V}}^{s} / \tan \alpha_{g}$ would lead to an error of approximately $10 \%$ since $\tan 30 / \tan 28 \approx 1.1$.

Putting the previous discussion into a modelling perspective, the following conclusions can be drawn:

(a) momentum fluxes over the suction plane and the injection plane are dominated by inviscid contributions. Wall friction is not the first-order effect for permeable plates, at least when the blowing ratio is not very small. Note however that the viscous contribution through wall friction is small but not negligible,

(b) vertical and spanwise momentum fluxes can easily be estimated and do not demand any modelling effort, at least as long as the main flows are aligned with the aperture midplane,

(c) regarding the injection side of the plate, the non-viscous streamwise momentum flux can be approximated by:

$$
-\rho \int_{S_{h}} \overline{U V}^{t} n_{2} d s \approx \frac{\dot{m}_{h}^{2}}{\rho \tan \alpha_{g}}
$$

where $\dot{m}_{h}$ is the mass flow rate per unit area in the hole, viz. $\dot{m}_{h}=\left(1 / S_{h}\right) \int_{S_{h}} \rho \bar{V}^{t} d s$. This approximation leads to an error of $18 \%$. The difference is mainly due to the two assumptions that $\alpha$ can be assessed by $\alpha_{g}$ and $\overline{U V}^{s}$ by ${\overline{\bar{U}^{t}}}^{s}{\overline{\bar{V}^{t}}}^{s}$ at the hole outlet. In order to improve the assessment of the inviscid contribution to the streamwise momentum flux on the injection side, one should improve these two assumptions,

(d) at the suction side of the plate, the flow direction is not controlled by the hole orientation. Instead, it is directly related to the streamwise velocity in the cold crossflow. Approximating the non-viscous streamwise momentum flux at the suction side by:

$$
-\rho \int_{S_{h}} \overline{U V}^{t} n_{2} d s \approx U_{2} \dot{m_{h}}
$$


where $U_{2}$ is the crossflow velocity in the casing side leads to an error of $20 \%$. The two assumptions $\overline{\overline{U V}}^{s} \approx \overline{\bar{U}}^{s} \overline{\bar{V}}^{s}$ and ${\overline{\bar{U}^{t}}}^{s} \approx U_{2}$ leads each to $10 \%$ error.

Of course, since only one operating point has been considered, there is no proof of the generality of these conclusions. For example, a multi-perforated plate whose thickness is less than approximately $0.6 d$ may behave very differently from thicker plates because the angle of the jets seen by the primary flow might be significantly different from the geometrical angle. However, since the geometrical (hole angle, diameter-to-thickness ratio) and flow (hole Reynolds number, injection parameter) characteristics considered in this paper are relevant to practical film cooling applications in gas turbines, one can expect that the above conclusions can serve as a guide for further developments. In other words, if the quantitative assessments of the different contributions of the wall fluxes presented in tables 5,6 and 7 are not universal, it is fair to believe that the trends reported are valid for practical FCFC applications.

\section{Conclusion}

A numerical methodology is proposed to generate a synthetic turbulent flow with effusion. The method presented simulates the flow around a perforated plate using a single-hole, bi-periodic domain, thus representing the interaction between a large (infinite) number of jets and the main streams. Such a periodic flow allows use of a refined mesh in a reduced computational domain to learn about the small-scale structure of the flow in the case of full-coverage film cooling. Both sides of the liner are computed to avoid any erroneous assumption regarding the flow in the aperture. The influence of the computational domain size is discussed by comparison of simulations based on 1-hole and 4-hole computational domains: time-averaged and root mean square velocity profiles as well as two-point correlations are compared and no major difference is observed. This important result allows regarding the 1-hole domain computations as reference simulations which can be used to generate a numerical database of effusion cooling flows. Quantitative comparisons are proposed with experimental results in the case of a large-scale isothermal configuration in order to precise the similarities and differences between a bi-periodic effusion flow over an infinite perforated plate and a spatially evolving configuration. Overall, the global structure of the flow is not modified and the simulations show good general agreement with the experimental results. However, appreciable differences are observed on the mean streamwise velocity. This is consistent with the observation, in spatially evolving effusion cooling film, that the mean streamwise velocity evolves from one row to the other, at least at the beginning of the plate. This is the main difference between a spatially evolving effusion cooling film and the synthetic flow presented in the paper.

The following main flow structures have been observed by investigating the suction, aperture and injection regions of the flow domain:

(a) Counter-rotating vortical structures inside and outside of the hole,

(b) Horseshoe vortex upstream of the jet and downstream spiral separation node vortices,

(c) Jetting effect with concentration of momentum along the upstream wall inside the hole,

(d) Separation at the entry and at the outlet of the hole due to high enough blowing ratio,

(e) Entrainment phenomenon in the wake of the jet, 
(f) Two streamwise counter-rotating vortices created at the lateral edges of the hole inlet on the suction side.

Regarding the instantaneous vortical structure of the flow, it is found that the vortices present in the windward shear layer are triggered by the in-hole vortices initiated at the downstream edge of the aspiration hole section.

From the obtained results, several key statements relevant to future modelling efforts can be made:

(a) The flow is highly inhomogeneous in the casing and the combustion chamber sides of the liner, as well as in the hole itself,

(b) A strong coupling is observed between the different parts of the computational domain,

(c) The jet is not aligned with the hole direction except in a small part of the hole,

(d) On both sides of the liner, the overall contribution of the wall shear stress over the solid plate is approximately $10 \%$ of the non-viscous flux due to the injection through the hole.

(e) A first-order model can be derived by estimating the streamwise momentum fluxes at both sides of the plate, assuming constant velocity profiles over the aperture inlet/outlet.

It is anticipated that these results will be useful in supporting future modelling efforts to account for multi-perforated plates in full-scale combustion chamber calculations.

The authors are grateful to the European Community for funding this work under the project INTELLECT-DM (Contract No. FP6 - AST3 - CT - 2003 - 502961), and to the BSC (Barcelona Supercomputing Center) and the CINES (Centre Informatique National pour l'Enseignement Supérieur) for the access to supercomputer facilities. A substantial part of this study was performed during the 2006 CTR Summer Program at Stanford. M. Shoeybi and Prof. G. Iaccarino are also gratefully acknowledged for performing the simulation equivalent to Run B with the CDP code. The authors would also like to thank Turbomeca and Dr. P. Miron for the access to the LARA experimental database.

\section{REFERENCES}

Ammari, H. D., Hay, N. \& LAmpard, D. 1990 The effect of density ratio on the heat transfer coefficient from a film-cooled flat plate. ASME J. Turbomach. 112, 444-450.

Andreopoulos, J. \& Rodi, W. 1984 Experimental investigation of jets in a crossflow. J. Fluid Mech. 138, 93-127.

Bazdidi-Tehrani, F. \& Andrews, G. E. 1994 Full-coverage discrete hole film cooling : investigation of the effect of variable density ratio. J. of Engineering for Gas Turbines and Power 116, 587-596.

Bergeles, G., Gosman, A. D. \& Launder, B. E. 1976 The near-field character of a jet discharged normal to a main stream. J. of Heat Transfer pp. 373-378.

Bergeles, G., Gosman, A. D. \& Launder, B. E. 1977 Near-field character of a jet discharged through a wall at $30 \mathrm{deg}$ to a mainstream. AIAA J. 15 (4), 499-504.

Brundage, A. L., Plesniak, M. W. \& Ramadhyani, S. 1999 Influence of coolant feed direction and hole length on film cooling jet velocity profiles. ASME Paper 99-GT-035 .

Champion, J.-L., Di Martino, P. \& Coron, X. 2005 Influence of flow characteristics on the discharge coefficient of a multiperforated wall. In Turbo Expo 2005, Reno Hilton, Reno Tahoe, Nevada USA, June 6-9 2005, , vol. GT2005-68904.

Cho, H. H. \& Goldstein, R. J. 1995a Heat (mass) transfer and film cooling effectiveness with injection through discrete holes: Part i-within holes and on the back surface. ASME J. Turbomach. 117, 440-450.

Cho, H. H. \& Goldstein, R. J. $1995 b$ Heat (mass) transfer and film cooling effectiveness with 
injection through discrete holes: Part ii-on the exposed surface. ASME J. Turbomach. 117, 451-460.

Colin, O. \& Rudgyard, M. 2000 Development of high-order Taylor-Galerkin schemes for unsteady calculations. J. Comp. Physics 162 (2), 338-371.

Cortelezzi, L. \& Karagozian, A. R. 2001 On the formation of the counter-rotating vortex pair in transverse jets. J. Fluid Mech. 446, 347-373.

Crawford, M. E., Kays, W. M. \& Moffat, R. J. 1980 Full-coverage film cooling. part i : Comparison of heat transfer data for three injection angles. J. of Engineering for Power 102, 1000-1005.

Dorignac, E., Vullierme, J. J., Broussely, M., Foulon, C. \& Mokkadem, M. 2005 Experimental heat transfer on the windward surface of a perforated flat plate. Int. J. of Thermal Sciences 44, 885-893.

Eriksen, V. L. \& Goldstein, R. J. 1974 Heat transfer and film cooling following injection through inclined circular tubes. J. of Heat Transfer 96, 239-245.

Errera, M. P. \& Chemin, S. 2004 A fluid-solid thermal coupling applied to an effusion cooling system. In 34th Fluid Dynamics Conference and Exhibit. Portland, Oregon.

Fric, T.F. \& Roshko, A. 1994 Vortical structure in the wake of a transverse jet. J. Fluid Mech. 279, 1-47.

Goldstein, R. J. 1971 Advances in Heat Transfer. Academic Press, New-York and London.

Gritsch, M., Schultz, A. \& WitTig, S. 2001 Effect of crossflows on the discharge coefficient of film cooling holes with varying angles of inclination and orientation. ASME J. Turbomach. 123, 781-787.

Gustafsson, K. M. B. 2001 Experimental studies of effusion cooling. PhD thesis, Chalmers University of Technology. Göteborg.

Hale, C. A., Plesniak, M. W. \& Ramadhyani, S. 2000 Structural features and surface heat transfer associated with a row of short-hole jets in crossflow. Int. Journal of Heat and Fluid Flow 21, 542-553.

HAM, F. \& IACCARINO, G. 2004 Energy conservation in collocated discretization schemes on unstructured meshes. In Annual Research Briefs 2004, 3-14. Center for Turbulence Research, NASA Ames/Stanford Univ.

Harrington, M. K, McWaters, M. A., Bogard, D. G., A., Lemmon C. \& Thole, K. A. 2001 Full-coverage film cooling with short normal injection holes. ASME TURBOEXPO 2001. 2001-GT-0130.

Hunt, J. C. R., Wray, A. A. \& Moin, P. 1988 Eddies, streams, and convergence zones in turbulent flows. In Proc. Summer Program CTR, NASA Ames - Stanford University.

IourokinA, I. V. \& LELE, S. K. 2006 Large eddy simulation of film-cooling above the flat surface with a large plenum and short exit holes. In 44th Aerospace Sciences Meeting and Exhibit.

Ivanov, Y. V. 1963 Shape of the centerline of an axisymmetric fan type jet in a cross flow. Izv. VUZ Aviotsionnaya Teknika 4.

Kelso, R. M., Lim, T. T. \& Perry, A. E. 1996 An experimental study of round jets in cross-flow. J. Fluid Mech. 306, 111-144.

Lefebvre, A. H. 1999 Gas Turbines Combustion. Taylor \& Francis.

Leylek, J. H. \& ZeRKLe, R. D. 1994 Discrete-jet film cooling: A comparison of computational results with experiments. ASME J. Turbomach. 116, 358-368.

MacManus, D. G. \& Eaton, J. A. 2000 Flow physics of discrete boundary layer suction measurements and predictions. J. Fluid Mech. 417, 47-75.

Margason, R. J. 1968 The path of a jet directed at large angles to a subsonic free stream. TN D-4919. NASA.

Margason, R. J. 1993 Fifty years of jet in crossflow research. In Computational and Experimental Assessment of Jets in Crossflow (ed. UK Winchester), , vol. AGARD-CP-534, pp. $1-41$.

Mayle, R.E. \& Camarata, F.J. 1975 Multihole cooling effectiveness and heat transfer. Journal of Heat Transfer 97, 534-538.

Mendez, S., Eldredge, J. D., Nicoud, F., Poinsot, T., Shoeybi, M. \& Iaccarino, G. 2006 Numerical investigation and preliminary modeling of a turbulent flow over a multiperforated plate. In Proc. Summer Program CTR, NASA Ames - Stanford University. 
Mendez, S. \& Nicoud, F. 2007 Numerical investigation of an anisothermal turbulent flow with effusion. In 5th International Symposium on Turbulence and Shear Flow Phenomena, pp. 791-796.

Mendez, S., Nicoud, F. \& Miron, P. 2005 Direct and large-eddy simulations of a turbulent flow with effusion. In ERCOFTAC WORKSHOP. Direct and Large-Eddy Simulations 6. Poitiers FRANCE.

Metzger, D. E., Takeuchi, D. I. \& Kuenstler, P. A. 1973 Effectiveness and heat transfer with full-coverage film-cooling. ASME paper .

Miron, P. 2005 Étude expérimentale des lois de parois et du film de refroidissement produit par une zone multiperforée sur une paroi plane. PhD thesis, Université de Pau et des Pays de l'Adour.

Miron, P., Bérat, C. \& Sabelnikov, V. 2004 Effect of blowing rate on the film cooling coverage on a multi-holed plate: application on combustor walls. In Eighth International Conference on Heat Transfer. Lisbon, Portugal.

Moin, P. \& MAhesh, K. 1998 Direct numerical simulation: A tool in turbulence research. Annu. Rev. Fluid Mech. 30 (539-578).

Moureau, V., Lartigue, G., Sommerer, Y., Angelberger, C., Colin, O. \& Poinsot, T. 2005 Numerical methods for unsteady compressible multi-component reacting flows on fixed and moving grids. J. Comp. Physics 202 (2), 710-736.

Muppidi, S. \& MAHESh, K. 2007 Direct numerical simulation of round turbulent jets in crossflow. J. Fluid Mech. 574, 59-84.

Nicoud, F. \& Ducros, F. 1999 Subgrid-scale stress modelling based on the square of the velocity gradient tensor. Flow, Turbulence and Combustion 62 (3), 183-200.

Papanicolaou, E., Giebert, D., Koch, R. \& Schultz, A. 2001 A conservation-based discretization approach for conjugate heat transfer calculations in hot-gas ducting turbomachinery components. International Journal of Heat and Mass Transfer 44, 3413-3429.

Peet, Y. V. 2006 Film cooling from inclined cylindrical holes using Large-Eddy Simulations. $\mathrm{PhD}$ thesis, Stanford University.

Peterson, S. D. \& Plesniak, M. W. 2002 Short-hole jet-in-crossflow velocity field and its relationship to film-cooling performance. Exps. Fluids 33, 889-898.

Peterson, S. D. \& Plesniak, M. W. $2004 a$ Evolution of jets emanating from short holes into crossflow. J. Fluid Mech. 503, 57-91.

Peterson, S. D. \& Plesniak, M. W. $2004 b$ Surface shear stress measurements around multiple jets in crossflow using the fringe imaging skin friction technique. Exps. Fluids 37, 497-503.

Pietrzyk, J. R., Bogard, D. G. \& Crawford, M. E. 1989 Hydrodynamic measurements of jets in crossflow for gas turbine film cooling applications. ASME J. Turbomach. 111, 139-145.

Piomelli, U., Ferziger, J. H., Moin, P. \& Kim, J. 1989 New approximate boundary conditions for large eddy simulations of wall-bounded flows. Phys. Fluids A 1 (6), 1061-68.

Prière, C., Gicquel, L. Y. M., Gajan, P., Strzelecki, A., Poinsot, T. \& Bérat, C. 2005 Experimental and numerical studies of dilution systems for low emission combustors. AIAA J. 43 (8), 1753-1766.

Prière, C., Gicquel, L. Y. M., Kaufmann, A., Krebs, W. \& Poinsot, T. 2004 LeS predictions of mixing enhancement for jets in cross-flows. J. of Turbulence 5, 005.

Renze, P., Meinke, M. \& SchröDer, W. 2006 LES of turbulent mixing in film cooling flows. In Conference on Turbulence and Interactions TI2006, May 29 - June 2, 2006, Porquerolles, France.

Rouvreau, S. 2001 Étude expérimentale de la structure moyenne et instantanée d'un film produit par une zone multiperforée sur une paroi plane. application au refroidissement des chambres de combustion des moteurs aéronautiques. PhD thesis, E.N.S.M.A. et Faculté des Sciences Fondamentales et Appliquées.

Schlüter, J. U. \& SchöNfEld, T. 2000 LES of jets in crossflow and its application to a gas turbine burner. Flow, Turbulence and Combustion 65 (2), 177-203.

Schmitt, P., Poinsot, T., Schuermans, B. \& Geigle, K. 2007 Large-eddy simulation and experimental study of heat transfer, nitric oxide emissions and combustion instability in a swirled turbulent high-pressure burner. J. Fluid Mech. 570, 17-46. 
Schönfeld, T. \& Rudgyard, M. 1999 Steady and unsteady flows simulations using the hybrid flow solver AVBP. AIAA J. $\mathbf{3 7}$ (11), 1378-1385.

Simpson, R. L. 1970 Characteristics of turbulent boundary layers at low reynolds numbers with and without transpiration. J. Fluid Mech. 42 (4), 769-802.

Sinha, A. K., Bogard, D. G. \& Crawford, M. E. 1991 Film-cooling effectiveness downstream of a single row of holes with variable density ratio. ASME J. Turbomach. 113, 442-449.

Smith, S. H. \& Mungal, M. G. 1998 Mixing, structure and scaling of the jet in crossflow. J. Fluid Mech. 357, 83-122.

Staffelbach, G., Gicquel, L. Y. M. \& Poinsot, T. 2006 Highly parallel large eddy simulations of multiburner configurations in industrial gas turbines. Lecture Notes in Computational Science and Engineering - Complex effects in Large Eddy Simulations 56, 325-336.

Thompson, K. W. 1990 Time dependent boundary conditions for hyperbolic systems. J. Comp. Physics 89, 439-461.

Tyagi, M. \& Acharya, S. 2003 Large eddy simulation of film cooling flow from an inclined cylindrical jet. ASME J. Turbomach. 125, 734-742.

Walters, D.K. \& LeYlek, J.H. 2000 A detailed analysis of film-cooling physics: Part 1streamwise injection with cylindrical holes. ASME J. Turbomach. 122, 102-112.

Yavuzkurt, S., Moffat, R. J. \& Kays, W. M. 1980 a Full coverage film cooling. Part 1. Three-dimensional measurements of turbulence structure. J. Fluid Mech. 101, 129-158.

Yavuzkurt, S., Moffat, R. J. \& Kays, W. M. $1980 b$ Full coverage film cooling. Part 2. Prediction of the recovery-region hydrodynamics. J. Fluid Mech. 101 (1), 159-178.

Yu, D., Ali, M. S. \& Lee, J. H. W. 2006 Multiple tandem jets in cross-flow. J. Hydr. Engrg 9, 971-982.

Yuan, L. L., Street, R. L. \& Ferziger, J. H. 1999 Large-eddy simulations of a round jet in crossflow. J. Fluid Mech. 379, 71-104. 